MATHEMATICS OF COMPUTATION

Volume 74, Number 250, Pages 521-542

S 0025-5718(04)01687-4

Article electronically published on July 20, 2004

\title{
ERROR ESTIMATES FOR SEMI-DISCRETE GAUGE METHODS FOR THE NAVIER-STOKES EQUATIONS
}

\author{
RICARDO H. NOCHETTO AND JAE-HONG PYO
}

\begin{abstract}
The gauge formulation of the Navier-Stokes equations for incompressible fluids is a new projection method. It splits the velocity $\mathbf{u}=\mathbf{a}+\nabla \phi$ in terms of auxiliary (nonphysical) variables a and $\phi$ and replaces the momentum equation by a heat-like equation for $\mathbf{a}$ and the incompressibility constraint by a diffusion equation for $\phi$. This paper studies two time-discrete algorithms based on this splitting and the backward Euler method for a with explicit boundary conditions and shows their stability and rates of convergence for both velocity and pressure. The analyses are variational and hinge on realistic regularity requirements on the exact solution and data. Both Neumann and Dirichlet boundary conditions are, in principle, admissible for $\phi$ but a compatibility restriction for the latter is uncovered which limits its applicability.
\end{abstract}

\section{The gauge or impulse FORMUlation}

Given an open bounded polyhedral domain $\Omega$ in $\mathbb{R}^{d}$, with $d=2$ or 3 , we consider the time-dependent Navier-Stokes equations of incompressible fluids:

$$
\begin{aligned}
\mathbf{u}_{t}+(\mathbf{u} \cdot \nabla) \mathbf{u}+\nabla p-\mu \Delta \mathbf{u} & =\mathbf{f}, & & \text { in } \Omega, \\
\operatorname{div} \mathbf{u} & =0, & & \text { in } \Omega, \\
\mathbf{u}(0, \mathbf{x}) & =\mathbf{u}^{0}, & & \text { in } \Omega,
\end{aligned}
$$

with vanishing Dirichlet boundary condition $\mathbf{u}=\mathbf{0}$ on $\partial \Omega$ and pressure mean-value $\int_{\Omega} p=0$. The primitive variables are the (vector) velocity $\mathbf{u}$ and the (scalar) pressure $p$. The viscosity $\mu=R e^{-1}$ is the reciprocal of the Reynolds number $R e$. Hereafter, vectors are denoted in boldface.

Pressure $p$ can be viewed in (1.1) as a Lagrange multiplier corresponding to the incompressibility condition $\operatorname{div} \mathbf{u}=0$. This coupling is responsible for compatibility conditions between the spaces for $\mathbf{u}$ and $p$, characterized by the celebrated inf-sup condition, and associated numerical difficulties [1,9]. On the other hand, projection methods were introduced independently by Chorin [3] and Temam [22, 24] in the late 1960s to decouple $\mathbf{u}$ and $p$ and thus reduce the computational cost. However, projection methods impose an artificial boundary condition on $p$, which leads to boundary layers and reduced convergence rates for $p$ [8, 20]. Error estimates can be found in [2, 8, 21].

Received by the editor March 21, 2003 and, in revised form, October 20, 2003.

2000 Mathematics Subject Classification. Primary 65M12, 65M15, 76D05.

Key words and phrases. Projection method, Gauge method, Navier-Stokes equation, incompressible fluids.

The first author was partially supported by NSF Grants DMS-9971450 and DMS-0204670.

The second author was partially supported by NSF Grant DMS-9971450. 
The gauge (or impulse) formulation, introduced by Oseledets [17] and E and Liu [6, 7], is a projection method especially conceived to cope with these inconsistencies. The gauge formulation consists of rewriting (1.1) in terms of two auxiliary variables, the vector field $\mathbf{a}$ and the scalar field $\phi$ (gauge variable), which satisfy $\mathbf{u}=\mathbf{a}+\nabla \phi$. Upon replacing this relation into the momentum equation in (1.1), we get

$$
\mathbf{a}_{t}+(\mathbf{u} \cdot \nabla) \mathbf{u}+\nabla\left(\phi_{t}-\mu \Delta \phi\right)+\nabla p-\mu \Delta \mathbf{a}=\mathbf{f} .
$$

Imposing

$$
p=-\phi_{t}+\mu \Delta \phi,
$$

we end up with the gauge formulation of (1.1) due to E and Liu [7]:

$$
\begin{aligned}
\mathbf{a}_{t}+(\mathbf{u} \cdot \nabla) \mathbf{u}-\mu \Delta \mathbf{a}=\mathbf{f}, & \text { in } \Omega, \\
-\Delta \phi=\operatorname{div} \mathbf{a}, & \text { in } \Omega, \\
\mathbf{u}=\mathbf{a}+\nabla \phi, & \text { in } \Omega, \\
p=-\phi_{t}+\mu \Delta \phi, & \text { in } \Omega .
\end{aligned}
$$

Suitable boundary conditions must be given to close this system. A key advantage of the gauge formulation is that no boundary condition is imposed on $p$ and that we are free to choose a convenient boundary condition for the nonphysical variable $\phi$ which, in view of (1.2), is expected to be smoother than $p$. We could take a homogeneous condition either of Neumann or Dirichlet type for $\phi$. To enforce the boundary condition $\mathbf{u}=\mathbf{0}$, we could either prescribe

$$
\partial_{\boldsymbol{\nu}} \phi=0, \quad \mathbf{a} \cdot \boldsymbol{\nu}=0, \quad \mathbf{a} \cdot \boldsymbol{\tau}=-\partial_{\boldsymbol{\tau}} \phi
$$

or

$$
\phi=0, \quad \mathbf{a} \cdot \boldsymbol{\nu}=-\partial_{\boldsymbol{\nu}} \phi, \quad \mathbf{a} \cdot \boldsymbol{\tau}=0,
$$

where $\boldsymbol{\nu}$ and $\boldsymbol{\tau}$ are the unit vectors in the normal and tangential directions, respectively. We call (1.4) the Neumann formulation and (1.5) the Dirichlet formulation.

Wang and Liu show, for the backward Euler time discretization of (1.3), that the order of convergence for velocity is 1 for the Neumann formulation and $\frac{1}{2}$ for the Dirichlet formulation [26]. Since [26] is based on asymptotic analysis, the exact solutions are assumed to be sufficiently smooth, a rather strong and unrealistic regularity requirement, particularly so for $t \downarrow 0$ for this entails nonlocal compatibility conditions between the initial data 11]; see Assumption A4 below. In addition, 26] does not address the convergence of pressure, which is the most sensitive variable. We use, instead, a variational technique to get rates of convergence for both velocity and pressure under realistic regularity assumptions on data. A distinctive aspect of our study is the assessment of pressure convergence. Since pressure is obtained through differentiation of $\phi$, the boundary conditions (1.4) and (1.5) play a central role. The Neumann condition (1.4) always leads to an optimal convergence rate for velocity and a suboptimal one for pressure. In contrast, the Dirichlet condition (1.5) fails to yield convergence of pressure and solely gives a reduced convergence rate for velocity. The error estimates are similar to those known for the Chorin method [18, [20, 21] and do not fully explain the advantages of the gauge methods [6, 7, 14, 15, 19]. They provide, however, a flexible methodology that extends to space discretization [14], a rare and fortunate situation for projection methods.

The paper is organized as follows. In \$2 we formulate a semi-implicit timediscrete gauge algorithm based on Neumann condition (1.4). In \$3] we introduce 
basic assumptions and recall regularity results for (1.1). We show in that the semi-implicit algorithms are unconditionally stable in energy norms, and so applicable for large Reynolds numbers; we also examine the explicit treatment of convection which requires a CFL condition. We give a priori error analyses in $\$ 5$ for velocity and its time derivative via energy techniques; we refer to 11, 25] for details. We present an a priori error analysis for pressure in $\$ 6$ We finally conclude in $\$ 7$ with a brief discussion of the Dirichlet condition.

\section{The gauge method with Neumann COndition}

We consider the backward Euler time discretization with uniform time step $\tau$ of gauge formulation (1.3) with Neumann (1.4) condition. In order to decouple the calculation of $\mathbf{a}^{n+1}$ and $\phi^{n+1}$ at time step $n+1$, it is necessary to extrapolate the boundary conditions from the previous time step. This extrapolation is responsible for a boundary layer. Note that $\tau$ indicates a tangential unit vector to $\partial \Omega$ whereas $\tau$ designates the time step. No confusion ever arises.

Algorithm 1 (Gauge method with Neumann condition (1.4)). Start with initial values $\phi^{0}=0$ and $\mathbf{a}^{0}=\mathbf{u}^{0}=\mathbf{u}(0, \mathbf{x})$. Repeat for $1 \leq n \leq N$

Step 1: Find $\mathbf{a}^{n+1}$ as the solution of

$$
\begin{aligned}
\frac{\mathbf{a}^{n+1}-\mathbf{a}^{n}}{\tau}+\left(\mathbf{u}^{n} \cdot \nabla\right) \mathbf{a}^{n+1}+\left(\mathbf{u}^{n} \cdot \nabla\right) \nabla \phi^{n}-\mu \Delta \mathbf{a}^{n+1}=\mathbf{f}\left(t^{n+1}\right), & \text { in } \Omega, \\
\mathbf{a}^{n+1} \cdot \boldsymbol{\nu}=0, \quad \mathbf{a}^{n+1} \cdot \boldsymbol{\tau}=-\partial_{\boldsymbol{\tau}} \phi^{n}, & \text { on } \partial \Omega .
\end{aligned}
$$

Step 2: Find $\phi^{n+1}$ as the solution of

$$
\begin{aligned}
-\Delta \phi^{n+1} & =\operatorname{div} \mathbf{a}^{n+1}, & & \text { in } \Omega, \\
\partial_{\boldsymbol{\nu}} \phi^{n+1} & =0, & & \text { on } \partial \Omega .
\end{aligned}
$$

Step 3: Update $\mathbf{u}^{n+1}$ :

$$
\mathbf{u}^{n+1}=\mathbf{a}^{n+1}+\nabla \phi^{n+1}, \quad \text { in } \Omega .
$$

Remark 2.1 (Pressure). One may compute the pressure whenever necessary as

$$
p^{n+1}=-\frac{\phi^{n+1}-\phi^{n}}{\tau}+\mu \Delta \phi^{n+1} .
$$

Remark 2.2 (Velocity boundary condition). In view of the extrapolated boundary condition $\mathbf{a}^{n+1} \cdot \boldsymbol{\tau}=-\partial_{\boldsymbol{\tau}} \phi^{n}$, the boundary condition of velocity $\mathbf{u}^{n+1}$ is

$$
\mathbf{u}^{n+1} \cdot \boldsymbol{\nu}=0, \quad \mathbf{u}^{n+1} \cdot \boldsymbol{\tau}=\partial_{\boldsymbol{\tau}} \phi^{n+1}-\partial_{\boldsymbol{\tau}} \phi^{n} \quad \text { on } \partial \Omega
$$

thus $\mathbf{u}^{n+1} \cdot \boldsymbol{\tau}$ is not zero, which represents a boundary layer effect. In order to reduce the boundary layer in (2.3), we can use the 2 nd order extrapolation formula $\mathbf{a}^{n+1} \cdot \boldsymbol{\tau}=-2 \partial_{\boldsymbol{\tau}} \phi^{n}+\partial_{\boldsymbol{\tau}} \phi^{n-1}[7]$.

Algorithm 1 is shown to be unconditionally stable in $\$$ which extends its applicability to large Reynolds numbers. We note that the equation (2.1) for $\mathbf{a}^{n+1}$ is still linear but nonsymmetric. It becomes symmetric upon treating the convection term explicitly in the momentum equation [6, 7, 26], namely,

$$
\frac{\mathbf{a}^{n+1}-\mathbf{a}^{n}}{\tau}+\left(\mathbf{u}^{n} \cdot \nabla\right) \mathbf{u}^{n}-\mu \Delta \mathbf{a}^{n+1}=\mathbf{f}\left(t^{n+1}\right) .
$$

The gauge algorithm based on (2.4) is stable provided $C \tau \leq \mu$ and a rather strong, but customary, assumption is made on the discrete solution; see 4 . 


\section{Preliminaries}

This section is mainly devoted to stating assumptions, reviewing some wellknown lemmas, and to proving basic properties of (1.1). The basic mathematical theory summarized here can be found in the works of Constantin and Foias [4], Heywood and Rannacher [11, and A. Prohl [18.

Let $H^{s}(\Omega)$ be the Sobolev space with $s$ derivatives in $L^{2}(\Omega), \mathbf{L}^{2}(\Omega)=\left(L^{2}(\Omega)\right)^{d}$ and $b f H^{s}(\Omega)=\left(H^{s}(\Omega)\right)^{d}$, where $d=2,3$. Let $\|\cdot\|_{0}$ denote the $\mathbf{L}^{2}(\Omega)$ norm, and $\langle\cdot, \cdot\rangle$ the corresponding inner product. Let $\|\cdot\|_{s}$ denote the norm of $H^{s}(\Omega)$ for $s \in \mathbb{R}$.

In the proof of convergence, we resort to a duality argument via the following Stokes equations:

$$
\begin{aligned}
-\Delta \mathbf{v}+\nabla q=\mathbf{g}, & \text { in } \Omega, \\
\operatorname{div} \mathbf{v}=0, & \text { in } \Omega, \\
\mathbf{v}=\mathbf{0}, & \text { on } \partial \Omega .
\end{aligned}
$$

We start with three basic assumptions about data $\Omega, \mathbf{u}^{0}, \mathbf{f}$ and solution $\mathbf{u}$.

Assumption A1 (Regularity of (3.1)). The unique solution $\{\mathbf{v}, q\}$ of the steady Stokes equation (3.1) satisfies

$$
\|\mathbf{v}\|_{2}+\|q\|_{1} \leq C\|\mathbf{g}\|_{0} .
$$

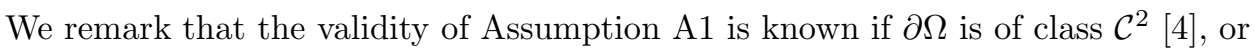
if $\Omega$ is a convex two-dimensional polygon [12] or a three-dimensional polyhedral [5].

Assumption A2 (Data regularity). The initial velocity $\mathbf{u}(0)$ and the forcing term in (1.1) satisfy

$$
\mathbf{u}(0) \in b f H^{2}(\Omega) \cap\left\{\mathbf{v} \in b f H_{0}^{1}(\Omega) \mid \operatorname{div} \mathbf{v}=0\right\} \quad \text { and } \quad \mathbf{f}, \mathbf{f}_{t} \in L^{\infty}\left(0, T ; \mathbf{L}^{2}(\Omega)\right) .
$$

Assumption A3 (Regularity of the solution $\mathbf{u}$ ). There exists $M \in \mathbb{R}$ such that

$$
\sup _{t \in[0, T]}\|\nabla \mathbf{u}(t)\|_{0} \leq M \text {. }
$$

We note that Assumption $\mathrm{A} 3$ is always satisfied in two dimensions, whereas it is valid in three dimensions provided $\|\mathbf{f}\|_{L^{\infty}\left(0, T ; \mathbf{L}^{2}(\Omega)\right)}$ and $\left\|\mathbf{u}^{0}\right\|_{1}$ are sufficiently small 11.

Let us introduce the following space which includes the solution $\mathbf{v}$ of the Stokes system (3.1):

$$
\mathbf{Z}:=\left\{\mathbf{z} \in b f H_{0}^{1}(\Omega) \mid \operatorname{div} \mathbf{z}=0\right\} .
$$

Then $\mathbf{Z}$ is a closed subspace of $b f H_{0}^{1}(\Omega)$ [9], and its dual space $\mathbf{Z}^{*}$ is equipped with the norm

$$
\|\mathbf{f}\|_{\mathbf{Z}^{*}}:=\sup _{\substack{\mathbf{z} \in \mathbf{Z}(\Omega) \\ \mathbf{z} \neq 0}} \frac{\langle\mathbf{f}, \mathbf{z}\rangle}{\|\mathbf{z}\|_{1}} .
$$

The following lemma is easy to prove.

Lemma 3.1 (Norm equivalence). Let $(\mathbf{v}, q, \mathbf{g})$ be the functions in the Stokes system (3.1). Then there exist two positive constants $C_{1}, C_{2}$ such that

$$
C_{1}\|\mathbf{g}\|_{\mathbf{Z}^{*}} \leq\|\mathbf{v}\|_{1} \leq C_{2}\|\mathbf{g}\|_{\mathbf{Z}^{*}} .
$$


We now define the trilinear form $\mathfrak{N}$ associated with the convection term in (1.1)

$$
\mathfrak{N}(\mathbf{u}, \mathbf{v}, \mathbf{w}):=\int_{\Omega}(\mathbf{u} \cdot \nabla) \mathbf{v} \cdot \mathbf{w} d \mathbf{x}
$$

for which the following properties are well known $[9]$.

Lemma 3.2 (Properties of $\mathfrak{N}$ ). Let $\mathbf{u}, \mathbf{v}, \mathbf{w} \in b f H^{1}(\Omega)$ and $\operatorname{div} \mathbf{u}=0$. If

$$
\mathbf{u} \cdot \boldsymbol{\nu}=0 \quad \text { or } \quad \mathbf{v}=\mathbf{0} \quad \text { on } \partial \Omega,
$$

then

$$
\mathfrak{N}(\mathbf{u}, \mathbf{v}, \mathbf{w})=-\mathfrak{N}(\mathbf{u}, \mathbf{w}, \mathbf{v}) \quad \text { and } \quad \mathfrak{N}(\mathbf{u}, \mathbf{v}, \mathbf{v})=0 .
$$

The Sobolev imbedding lemma yields the following results, which will be used later in dealing with the convection term of (1.1).

Lemma 3.3 (Bounds on trilinear form). If $d \leq 4$, then

$$
\int_{\Omega} \mathbf{u} \cdot \mathbf{v} \cdot \mathbf{w} d \mathbf{x} \leq\left\{\begin{array}{l}
C\|\mathbf{u}\|_{0}\|\mathbf{v}\|_{1}\|\mathbf{w}\|_{1} \\
C\|\mathbf{u}\|_{2}\|\mathbf{v}\|_{0}\|\mathbf{w}\|_{0}
\end{array}\right.
$$

and if $d \leq 3$, then

$$
\int_{\Omega} \mathbf{u} \cdot \mathbf{v} \cdot \mathbf{w} d \mathbf{x} \leq C\|\mathbf{u}\|_{1}\|\mathbf{v}\|_{0}^{1 / 2}\|\mathbf{v}\|_{1}^{1 / 2}\|\mathbf{w}\|_{0} .
$$

Heywood and Rannacher proved the following a priori regularity estimates [11].

Lemma 3.4 (Uniform and weighted a priori estimates). Let $\sigma(t)$ be the weight function

$$
\sigma(t)=\min \{t, 1\} \text {. }
$$

Suppose Assumptions $A[1-A]$ hold, and let $0<T \leq \infty$. Then the solution $(\mathbf{u}, p)$ of (1.1) satisfies

$$
\sup _{0<t<T}\left(\|\mathbf{u}\|_{2}+\left\|\mathbf{u}_{t}\right\|_{0}+\|p\|_{1}\right) \leq M, \quad \int_{0}^{T}\left\|\mathbf{u}_{t}\right\|_{1}^{2} d t \leq M
$$

and

$$
\sup _{0<t<T}\left(\sigma(t)\left\|\mathbf{u}_{t}\right\|_{1}^{2}\right) \leq M, \quad \int_{0}^{T} \sigma(t)\left(\left\|\mathbf{u}_{t}\right\|_{2}^{2}+\left\|\mathbf{u}_{t t}\right\|_{0}^{2}+\left\|p_{t}\right\|_{1}^{2}\right) d t \leq M .
$$

The following nonlocal assumption is used to remove the weight $\sigma(t)$ in the error estimates for $\mathbf{u}_{t}$ of $\$ 5$ and the pressure of $₫[6$.

Assumption A4 (Nonlocal compatibility). Let $\mathbf{u}^{0}$ and $\mathbf{f}^{0}=\mathbf{f}(0, \cdot)$ be such that

$$
\left\|\nabla \mathbf{u}_{t}(0)\right\|_{0} \leq M
$$

Combining (3.8) with [11, Corollary 2.1], we realize that (3.7) is equivalent to the initial data $\mathbf{u}^{0}, p^{0}=p(0, \cdot), \mathbf{f}^{0}$ satisfying the overdetermined system

$$
\Delta p^{0}=\operatorname{div}\left(\mathbf{f}^{0}-\left(\mathbf{u}^{0} \cdot \nabla\right) \mathbf{u}^{0}\right) \quad \text { in } \Omega, \quad \nabla p^{0}=\Delta \mathbf{u}^{0}+\mathbf{f}^{0}-\left(\mathbf{u}^{0} \cdot \nabla\right) \mathbf{u}^{0} \quad \text { on } \partial \Omega .
$$

This is true if $\mathbf{u}^{0}=\mathbf{f}^{0}=\mathbf{0}$, in which case also $p^{0}=0$ and $\left\|\nabla \mathbf{u}_{t}(0)\right\|_{0}=0$. However, $\left\|\nabla \mathbf{u}_{t}(t)\right\|_{0}$ blows-up in general as $t \downarrow 0$, thereby uncovering the practical limitations of results based on higher regularity than (3.5) and (3.6) uniformly for $t \downarrow 0$. 
The proof of Corollary 2.1 in [11, which assumes $\mathbf{u}^{0}=\mathbf{f}^{0}=\mathbf{0}$ instead of (3.7), implies Lemma 3.5 below. Lemma 3.6 extends Lemma 3.5 to negative norms and is instrumental in $\$ 5$.

Lemma 3.5 (Uniform a priori estimates). Suppose Assumptions A1-A 3 hold and let $0<T \leq \infty$. Then (3.7) is valid if and only if

$$
\int_{0}^{T}\left\|\mathbf{u}_{t t}(t)\right\|_{0}^{2} d t+\sup _{0<t<T}\left\|\nabla \mathbf{u}_{t}(t)\right\|_{0}^{2} \leq M
$$

Furthermore, if (3.7) holds, then

$$
\int_{0}^{T}\left(\left\|p_{t}(t)\right\|_{1}^{2}+\left\|\mathbf{u}_{t}(t)\right\|_{2}^{2}\right) d t \leq M
$$

Lemma 3.6 (A priori estimates on $\mathbf{Z}^{*}$ ). If Assumptions A1-A 1 hold, then we have

$$
\int_{0}^{T}\left\|\mathbf{u}_{t t}(t)\right\|_{\mathbf{Z}^{*}}^{2} d t \leq M
$$

Furthermore, if (3.7) holds, then

$$
\sup _{0<t<T}\left\|\mathbf{u}_{t t}(t)\right\|_{\mathbf{Z}^{*}}^{2} \leq M .
$$

Proof. Since $\|\nabla q\|_{\mathbf{Z}^{*}}=0$ for all $q \in L^{2}(\Omega)$, differentiating the momentum equation with respect to $t$ and utilizing Lemmas $3.2+3.3$ yields

$$
\begin{aligned}
\left\|\mathbf{u}_{t t}\right\|_{\mathbf{Z}^{*}} & \leq\left\|\left(\mathbf{u}_{t} \cdot \nabla\right) \mathbf{u}\right\|_{\mathbf{Z}^{*}}+\left\|(\mathbf{u} \cdot \nabla) \mathbf{u}_{t}\right\|_{\mathbf{Z}^{*}}+\mu\left\|\Delta \mathbf{u}_{t}\right\|_{\mathbf{Z}^{*}}+\left\|\mathbf{f}_{t}\right\|_{\mathbf{Z}^{*}} \\
& \leq C\left(\|\mathbf{u}\|_{2}\left\|\mathbf{u}_{t}\right\|_{0}+\left\|\mathbf{f}_{t}\right\|_{0}\right)+C \mu\left\|\nabla \mathbf{u}_{t}\right\|_{0} .
\end{aligned}
$$

Invoking Lemmas $3.4 \sqrt{3.5}$ and Assumption A2, we easily obtain both (3.10) and (3.11).

The following elementary but crucial relation is derived in [13, 19, 23].

Lemma 3.7 (div-grad relation). If $\mathbf{v} \in$ bf $H_{0}^{1}(\Omega)$, then

$$
\|\operatorname{div} \mathbf{v}\|_{0} \leq\|\nabla \mathbf{v}\|_{0} \text {. }
$$

\section{Stability}

We prove now that Algorithm 1 is unconditionally stable. According to (2.3) the time-discrete function $\mathbf{u}^{n+1}$ does not vanish on the boundary $\partial \Omega$, and it is thus difficult to use $\mathbf{u}^{n+1}$ as a test function. To get around this issue, we introduce the auxiliary function $\widehat{\mathbf{u}}^{n+1}$ which vanishes on $\partial \Omega$ :

$$
\widehat{\mathbf{u}}^{n+1}:=\mathbf{a}^{n+1}+\nabla \phi^{n} .
$$

The following useful properties of $\widehat{\mathbf{u}}^{n+1}$ are rather easy to show.

Lemma 4.1 (Properties of $\widehat{\mathbf{u}}^{n}$ and $\mathbf{u}^{n}$ ). For all $n, m$ nonnegative integers, we have

$$
\begin{gathered}
\widehat{\mathbf{u}}^{n}=\mathbf{0}, \quad \text { on } \partial \Omega, \\
\widehat{\mathbf{u}}^{n+1}=\mathbf{a}^{n+1}+\nabla \phi^{n}=\mathbf{u}^{n+1}-\nabla\left(\phi^{n+1}-\phi^{n}\right), \\
\left\langle\mathbf{u}^{n}, \nabla \phi^{m}\right\rangle=0, \quad \text { and } \quad\left\langle\widehat{\mathbf{u}}^{n}, \mathbf{u}^{m}\right\rangle=\left\langle\mathbf{u}^{n}, \mathbf{u}^{m}\right\rangle .
\end{gathered}
$$


Theorem 4.2 (Stability). Algorithm 1 is unconditionally stable in the sense that for all $\tau>0$ the following a priori bound holds:

$$
\begin{aligned}
& \sum_{n=0}^{N}\left(\left\|\mathbf{u}^{n+1}-\mathbf{u}^{n}\right\|_{0}^{2}+2\left\|\nabla\left(\phi^{n+1}-\phi^{n}\right)\right\|_{0}^{2}+\frac{\mu \tau}{2}\left\|\nabla \widehat{\mathbf{u}}^{n+1}\right\|_{0}^{2}\right) \\
& +\left\|\mathbf{u}^{N+1}\right\|_{0}^{2}+\mu \tau\left\|\Delta \phi^{N+1}\right\|_{0}^{2} \leq\left\|\mathbf{u}^{0}\right\|_{0}^{2}+\frac{C \tau}{\mu} \sum_{n=0}^{N}\left\|\mathbf{f}\left(t^{n+1}\right)\right\|_{-1}^{2} .
\end{aligned}
$$

Proof. By definition of $\mathbf{u}^{n+1}$ and $\widehat{\mathbf{u}}^{n+1}$, the momentum equation (2.1) can be rewritten as follows:

$$
\frac{\widehat{\mathbf{u}}^{n+1}-\mathbf{u}^{n}}{\tau}+\left(\mathbf{u}^{n} \cdot \nabla\right) \widehat{\mathbf{u}}^{n+1}-\mu \Delta\left(\widehat{\mathbf{u}}^{n+1}-\nabla \phi^{n}\right)=\mathbf{f}\left(t^{n+1}\right) .
$$

We now multiply by $2 \tau \widehat{\mathbf{u}}^{n+1} \in b f H_{0}^{1}(\Omega)$ and use Lemmas 3.2 and 4.1 to get

$$
\begin{aligned}
\left\|\mathbf{u}^{n+1}\right\|_{0}^{2} & -\left\|\mathbf{u}^{n}\right\|_{0}^{2}+\left\|\mathbf{u}^{n+1}-\mathbf{u}^{n}\right\|_{0}^{2}+2\left\|\nabla\left(\phi^{n+1}-\phi^{n}\right)\right\|_{0}^{2}+2 \mu \tau\left\|\nabla \widehat{\mathbf{u}}^{n+1}\right\|_{0}^{2} \\
& =2 \mu \tau\left\langle\Delta \phi^{n}, \operatorname{div} \widehat{\mathbf{u}}^{n+1}\right\rangle+2 \tau\left\langle\mathbf{f}\left(t^{n+1}\right), \widehat{\mathbf{u}}^{n+1}\right\rangle=A_{1}+A_{2} .
\end{aligned}
$$

In view of Lemmas 3.7 and 4.1 we have $\left\|\Delta\left(\phi^{n+1}-\phi^{n}\right)\right\|_{0}^{2}=\left\|\operatorname{div} \widehat{\mathbf{u}}^{n+1}\right\|_{0}^{2} \leq$ $\left\|\nabla \widehat{\mathbf{u}}^{n+1}\right\|_{0}^{2}$, whence

$$
\begin{aligned}
A_{1} & =-2 \mu \tau\left\langle\Delta \phi^{n}, \Delta\left(\phi^{n+1}-\phi^{n}\right)\right\rangle \\
& =-\mu \tau\left(\left\|\Delta \phi^{n+1}\right\|_{0}^{2}-\left\|\Delta \phi^{n}\right\|_{0}^{2}-\left\|\Delta\left(\phi^{n+1}-\phi^{n}\right)\right\|_{0}^{2}\right) \\
& \leq-\mu \tau\left(\left\|\Delta \phi^{n+1}\right\|_{0}^{2}-\left\|\Delta \phi^{n}\right\|_{0}^{2}\right)+\mu \tau\left\|\nabla \widehat{\mathbf{u}}^{n+1}\right\|_{0}^{2} .
\end{aligned}
$$

Clearly,

$$
A_{2} \leq 2 \tau\left\|\mathbf{f}\left(t^{n+1}\right)\right\|_{-1}\left\|\widehat{\mathbf{u}}^{n+1}\right\|_{1} \leq \frac{C \tau}{\mu}\left\|\mathbf{f}\left(t^{n+1}\right)\right\|_{-1}^{2}+\frac{\tau \mu}{2}\left\|\nabla \widehat{\mathbf{u}}^{n+1}\right\|_{0}^{2} .
$$

Inserting $A_{1}-A_{2}$ back into (4.6) and summing over $n$ from 0 to $N$, we get (4.4).

If we treat the convection term explicitly, namely $\left(\mathbf{u}^{n} \cdot \nabla\right) \mathbf{u}^{n}$, then the momentum equation (2.4) becomes

$$
\frac{\widehat{\mathbf{u}}^{n+1}-\mathbf{u}^{n}}{\tau}+\left(\mathbf{u}^{n} \cdot \nabla\right) \mathbf{u}^{n}-\mu \Delta\left(\widehat{\mathbf{u}}^{n+1}-\nabla \phi^{n}\right)=\mathbf{f}\left(t^{n+1}\right), \quad \text { in } \Omega .
$$

In order to estimate $\left(\mathbf{u}^{n} \cdot \nabla\right) \mathbf{u}^{n}$, we need to assume that the semi-discrete solution $\mathbf{u}^{n}$ is bounded in $\mathbf{L}^{\infty}(\Omega)$. This is a customary but rather strong assumption [26], which is not required in Theorem 4.2 .

Remark 4.3 (Stability for explicit Algorithm 1). Suppose

$$
\max _{0 \leq n \leq N+1}\left\|\mathbf{u}^{n}\right\|_{\mathbf{L}^{\infty}(\Omega)} \leq M .
$$

If Assumptions A $1-\mathrm{A} 3$ hold, and the stability constraint $2 M^{2} \tau \leq \mu$ is enforced, then the following a priori estimate is valid:

$$
\left\|\mathbf{u}^{N+1}\right\|_{0}^{2}+\mu \tau\left\|\Delta \phi^{N+1}\right\|_{0}^{2}+\frac{\mu \tau}{4} \sum_{n=0}^{N}\left\|\nabla \widehat{\mathbf{u}}^{n+1}\right\|_{0}^{2} \leq\left\|\mathbf{u}^{0}\right\|_{0}^{2}+\frac{C}{\mu} \tau \sum_{n=0}^{N}\left\|\mathbf{f}\left(t^{n+1}\right)\right\|_{-1}^{2} .
$$




\section{ERROR ANALYSIS FOR VELOCITY}

In this section, we carry out the error analysis for velocity of Algorithm 1 We first prove that the convergence rate of velocity is of order $\frac{1}{2}$, and then we improve the rate to order 1 .

Let $\left(\mathbf{u}\left(t^{n+1}\right), p\left(t^{n+1}\right)\right)$ be the exact solution of 1.1 at the time step $t^{n+1}$. If $\left(\widehat{\mathbf{u}}^{n+1}, \mathbf{u}^{n+1}, p^{n+1}\right)$ is the solution of the Algorithm [1 then we denote the corresponding error by

$$
\widehat{\mathbf{E}}^{n+1}:=\mathbf{u}\left(t^{n+1}\right)-\widehat{\mathbf{u}}^{n+1}, \quad \mathbf{E}^{n+1}:=\mathbf{u}\left(t^{n+1}\right)-\mathbf{u}^{n+1}, \quad e^{n+1}:=p\left(t^{n+1}\right)-p^{n+1} .
$$

We observe again that $\widehat{\mathbf{u}}^{n+1}=\mathbf{0}$ on $\partial \Omega$ and $\operatorname{div} \widehat{\mathbf{u}}^{n+1} \neq 0$ in $\Omega$, whereas $\operatorname{div} \mathbf{u}^{n+1}=0$ in $\Omega$ and $\mathbf{u}^{n+1}=\nabla\left(\phi^{n+1}-\phi^{n}\right) \neq \mathbf{0}$ on $\partial \Omega$. The following lemma results directly from Lemma 4.1.

Lemma 5.1 (Properties of error functions). For all n, $m$ nonnegative integers, we have

$$
\begin{gathered}
\widehat{\mathbf{E}}^{n+1}=\mathbf{0}, \quad \text { on } \partial \Omega, \\
\operatorname{div} \mathbf{E}^{n+1}=0, \quad \widehat{\mathbf{E}}^{n+1}=\mathbf{E}^{n+1}+\nabla\left(\phi^{n+1}-\phi^{n}\right), \\
\left\langle\mathbf{E}^{n}, \nabla \phi^{m}\right\rangle=0, \quad \text { and } \quad\left\langle\widehat{\mathbf{E}}^{n}, \mathbf{E}^{m}\right\rangle=\left\langle\mathbf{E}^{n}, \mathbf{E}^{m}\right\rangle .
\end{gathered}
$$

Lemma 5.2 (Additional properties of error functions). We have

$$
\begin{gathered}
\left\|\Delta\left(\phi^{n+1}-\phi^{n}\right)\right\|_{0}^{2}=\left\|\operatorname{div} \widehat{\mathbf{E}}^{n+1}\right\|_{0}^{2} \leq\left\|\nabla \widehat{\mathbf{E}}^{n+1}\right\|_{0}^{2}, \\
\left\|\widehat{\mathbf{E}}^{n+1}\right\|_{0}^{2}=\left\|\mathbf{E}^{n+1}\right\|_{0}^{2}+\left\|\nabla\left(\phi^{n+1}-\phi^{n}\right)\right\|_{0}^{2}, \\
\left\|\mathbf{E}^{n+1}\right\|_{1}^{2} \leq C\left(\left\|\widehat{\mathbf{E}}^{n+1}\right\|_{1}^{2}+\left\|\phi^{n+1}-\phi^{n}\right\|_{2}^{2}\right) \leq C\left\|\nabla \widehat{\mathbf{E}}^{n+1}\right\|_{0}^{2} .
\end{gathered}
$$

Proof. It is a simple consequence of Assumptions A 1 and Lemmas 3.7 and 5.1

To examine the gauge algorithm, we first show that the semi-discrete solution $\mathbf{u}^{n+1}$ converges to $\mathbf{u}\left(t^{n+1}\right)$ in $L^{\infty}\left(0, T ; \mathbf{L}^{2}(\Omega)\right)$ with order $\frac{1}{2}$ (see Theorem 5.3 ). We then improve the rate of convergence to order 1 in $L^{2}\left(0, T ; \mathbf{L}^{2}(\Omega)\right)$ and $L^{\infty}\left(0, T ; \mathbf{Z}^{*}\right)$ in Theorem 5.7. The result of Theorem 5.3 is instrumental in deriving Theorem 5.7 .

Theorem 5.3 (Reduced rate of convergence for velocity). Let Assumptions $A[1-A 3$ hold. Then the velocity error functions satisfy

$$
\begin{aligned}
\sum_{n=0}^{N}\left(\left\|\mathbf{E}^{n+1}-\mathbf{E}^{n}\right\|_{0}^{2}\right. & \left.+\left\|\nabla\left(\phi^{n+1}-\phi^{n}\right)\right\|_{0}^{2}+\frac{\mu \tau}{2}\left\|\nabla \widehat{\mathbf{E}}^{n+1}\right\|_{0}^{2}\right) \\
& +\left\|\mathbf{E}^{N+1}\right\|_{0}^{2}+\mu \tau\left\|\Delta \phi^{N+1}\right\|_{0}^{2} \leq C \tau .
\end{aligned}
$$

Proof. By virtue of Taylor expansion for the exact velocity $\mathbf{u}(t)$, we get

$$
\begin{aligned}
\frac{\mathbf{u}\left(t^{n+1}\right)-\mathbf{u}\left(t^{n}\right)}{\tau} & +\left(\mathbf{u}\left(t^{n+1}\right) \cdot \nabla\right) \mathbf{u}\left(t^{n+1}\right) \\
& +\nabla p\left(t^{n+1}\right)-\mu \Delta \mathbf{u}\left(t^{n+1}\right)=\mathbf{R}^{n+1}+\mathbf{f}\left(t^{n+1}\right)
\end{aligned}
$$


where $\mathbf{R}^{n+1}:=\frac{1}{\tau} \int_{t^{n}}^{t^{n+1}}\left(t^{n}-t\right) \mathbf{u}_{t t}(t) d t$ is the truncation error. Subtracting (4.5) from (5.8) yields the error equation

$$
\begin{aligned}
\frac{\widehat{\mathbf{E}}^{n+1}-\mathbf{E}^{n}}{\tau}-\mu \Delta \widehat{\mathbf{E}}^{n+1} & =\mathbf{R}^{n+1}-\nabla p\left(t^{n+1}\right)+\mu \nabla \Delta \phi^{n} \\
& -\left(\mathbf{u}\left(t^{n+1}\right) \cdot \nabla\right) \mathbf{u}\left(t^{n+1}\right)+\left(\mathbf{u}^{n} \cdot \nabla\right) \widehat{\mathbf{u}}^{n+1} .
\end{aligned}
$$

Multiplying (5.9) by $2 \tau \widehat{\mathbf{E}}^{n+1} \in b f H_{0}^{1}(\Omega)$ and invoking Lemma 5.1 (5.9) becomes

$$
\begin{aligned}
& \left\|\mathbf{E}^{n+1}\right\|_{0}^{2}-\left\|\mathbf{E}^{n}\right\|_{0}^{2}+\left\|\mathbf{E}^{n+1}-\mathbf{E}^{n}\right\|_{0}^{2}+2\left\|\nabla\left(\phi^{n+1}-\phi^{n}\right)\right\|_{0}^{2} \\
& \quad+2 \mu \tau\left\|\nabla \widehat{\mathbf{E}}^{n+1}\right\|_{0}^{2}=2 \tau\left\langle\mathbf{R}^{n+1}, \widehat{\mathbf{E}}^{n+1}\right\rangle \\
& \quad+2 \tau\left\langle p\left(t^{n+1}\right), \operatorname{div} \widehat{\mathbf{E}}^{n+1}\right\rangle-2 \mu \tau\left\langle\Delta \phi^{n}, \operatorname{div} \widehat{\mathbf{E}}^{n+1}\right\rangle \\
& \quad-2 \tau\left(\mathfrak{N}\left(\mathbf{u}\left(t^{n+1}\right), \mathbf{u}\left(t^{n+1}\right), \widehat{\mathbf{E}}^{n+1}\right)-\mathfrak{N}\left(\mathbf{u}^{n}, \widehat{\mathbf{u}}^{n+1}, \widehat{\mathbf{E}}^{n+1}\right)\right)=\sum_{i=1}^{4} A_{i} .
\end{aligned}
$$

We now estimate terms $A_{1}$ to $A_{4}$ separately. Using Hölder inequality,

$$
\begin{aligned}
\left\|\mathbf{R}^{n+1}\right\|_{0}^{2} & \leq \frac{1}{\tau^{2}} \int_{t^{n}}^{t^{n+1}}\left(t-t^{n}\right) d t \int_{t^{n}}^{t^{n+1}}\left(t-t^{n}\right)\left\|\mathbf{u}_{t t}(t)\right\|_{0}^{2} d t \\
& \leq C \int_{t^{n}}^{t^{n+1}} \sigma(t)\left\|\mathbf{u}_{t t}(t)\right\|_{0}^{2} d t
\end{aligned}
$$

whence we deduce from (5.5)

$$
A_{1} \leq C \tau \int_{t^{n}}^{t^{n+1}} \sigma(t)\left\|\mathbf{u}_{t t}(t)\right\|_{0}^{2} d t+C \tau\left\|\mathbf{E}^{n+1}\right\|_{0}^{2}+\frac{1}{2}\left\|\nabla\left(\phi^{n+1}-\phi^{n}\right)\right\|_{0}^{2} .
$$

On employing Lemma 5.1 and the boundary values $\partial_{\boldsymbol{\nu}}\left(\phi^{n+1}-\phi^{n}\right)=0$, we obtain

$$
A_{2} \leq C \tau^{2}\left\|\nabla p\left(t^{n+1}\right)\right\|_{0}^{2}+\frac{1}{2}\left\|\nabla\left(\phi^{n+1}-\phi^{n}\right)\right\|_{0}^{2} .
$$

Making use of (5.2) and (5.4), we arrive at

$$
\begin{aligned}
A_{3} & =-2 \mu \tau\left\langle\Delta \phi^{n}, \Delta\left(\phi^{n+1}-\phi^{n}\right)\right\rangle \\
& =-\mu \tau\left(\left\|\Delta \phi^{n+1}\right\|_{0}^{2}-\left\|\Delta \phi^{n}\right\|_{0}^{2}\right)+\mu \tau\left\|\Delta\left(\phi^{n+1}-\phi^{n}\right)\right\|_{0}^{2} \\
& \leq-\mu \tau\left(\left\|\Delta \phi^{n+1}\right\|_{0}^{2}-\left\|\Delta \phi^{n}\right\|_{0}^{2}\right)+\mu \tau\left\|\nabla \widehat{\mathbf{E}}^{n+1}\right\|_{0}^{2} .
\end{aligned}
$$

To estimate the convection term $A_{4}$, we first note that $\mathfrak{N}\left(\mathbf{u}^{n}, \widehat{\mathbf{E}}^{n+1}, \widehat{\mathbf{E}}^{n+1}\right)=0$ by Lemma 3.2. We next invoke $\left\|\mathbf{u}\left(t^{n+1}\right)\right\|_{2} \leq M$, which comes from (3.5), and infer

$$
\begin{aligned}
A_{4}= & -2 \tau \mathfrak{N}\left(\mathbf{u}\left(t^{n+1}\right)-\mathbf{u}\left(t^{n}\right), \mathbf{u}\left(t^{n+1}\right), \widehat{\mathbf{E}}^{n+1}\right) \\
& -2 \tau \mathfrak{N}\left(\mathbf{E}^{n}, \mathbf{u}\left(t^{n+1}\right), \widehat{\mathbf{E}}^{n+1}\right)-2 \tau \mathfrak{N}\left(\mathbf{u}^{n}, \widehat{\mathbf{E}}^{n+1}, \widehat{\mathbf{E}}^{n+1}\right) \\
\leq & C \tau\left(\left\|\mathbf{u}\left(t^{n+1}\right)-\mathbf{u}\left(t^{n}\right)\right\|_{0}+\left\|\mathbf{E}^{n}\right\|_{0}\right)\left\|\mathbf{u}\left(t^{n+1}\right)\right\|_{2}\left\|\nabla \widehat{\mathbf{E}}^{n+1}\right\|_{0} \\
\leq & \frac{C \tau^{2}}{\mu} \int_{t^{n}}^{t^{n+1}}\left\|\mathbf{u}_{t}(t)\right\|_{0}^{2} d t+\frac{C \tau}{\mu}\left\|\mathbf{E}^{n}\right\|_{0}^{2}+\frac{\mu \tau}{2}\left\|\nabla \widehat{\mathbf{E}}^{n+1}\right\|_{0}^{2} .
\end{aligned}
$$


Replacing $A_{1}-A_{4}$ back into (5.10) and summing over $n$ from 0 to $N$ implies

$$
\begin{aligned}
& \left\|\mathbf{E}^{N+1}\right\|_{0}^{2}+\mu \tau\left\|\Delta \phi^{N+1}\right\|_{0}^{2}+\sum_{n=0}^{N}\left(\left\|\nabla\left(\phi^{n+1}-\phi^{n}\right)\right\|_{0}^{2}+\left\|\mathbf{E}^{n+1}-\mathbf{E}^{n}\right\|_{0}^{2}\right) \\
& +\frac{\mu \tau}{2} \sum_{n=0}^{N}\left\|\nabla \widehat{\mathbf{E}}^{n+1}\right\|_{0}^{2} \leq C \tau \sum_{n=0}^{N}\left(\frac{1}{\mu}\left\|\mathbf{E}^{n}\right\|_{0}^{2}+\left\|\mathbf{E}^{n+1}\right\|_{0}^{2}+\tau\left\|\nabla p\left(t^{n+1}\right)\right\|_{0}^{2}\right) \\
& +\frac{C \tau^{2}}{\mu} \int_{0}^{t^{N+1}}\left\|\mathbf{u}_{t}(t)\right\|_{0}^{2} d t+C \tau \int_{0}^{t^{N+1}} \sigma(t)\left\|\mathbf{u}_{t t}(t)\right\|_{0}^{2} d t .
\end{aligned}
$$

By the discrete Gronwall lemma and Lemma 3.4 we finally obtain (5.7).

Remark 5.4 (Dependence on $\mu$ ). The constant $C$ in (5.7) depends exponentially on $\mu$ which is the reciprocal of the Reynolds number. This is unfortunate but customary in the error analysis of 11.1, except perhaps for the pipe flow in [10].

Remark 5.5 (Estimates for initial errors). Choosing $N=0$ in (5.15), and realizing that $\mathbf{E}^{0}=\mathbf{0}$ and $\phi^{0}=0$, we readily have from Lemma 3.4 and Theorem 5.3

$$
\begin{aligned}
\left\|\mathbf{E}^{1}\right\|_{0}^{2} & +\left\|\nabla \phi^{1}\right\|_{0}^{2}+\mu \tau\left\|\Delta \phi^{1}\right\|_{0}^{2}+\frac{\mu \tau}{2}\left\|\nabla \widehat{\mathbf{E}}^{1}\right\|_{0}^{2} \leq \frac{C \tau}{\mu}\left(\left\|\mathbf{E}^{1}\right\|_{0}^{2}+\left\|\nabla \phi^{1}\right\|_{0}^{2}\right) \\
& +C \tau^{2}\left\|\nabla p\left(t^{1}\right)\right\|_{0}^{2}+\frac{C \tau^{2}}{\mu} \int_{0}^{t^{1}}\left\|\mathbf{u}_{t}(t)\right\|_{0}^{2} d t+C \tau \int_{0}^{t^{1}} \sigma(t)\left\|\mathbf{u}_{t t}(t)\right\|_{0}^{2} d t \\
& \leq C \tau^{2}+C \tau \int_{0}^{t^{1}} \sigma(t)\left\|\mathbf{u}_{t t}(t)\right\|_{0}^{2} d t \leq C \tau,
\end{aligned}
$$

or $\leq C \tau^{2}$ provided Assumption A4also holds because then (3.8) applies and $\sigma(t) \leq$ $\tau$ for $t \leq t^{1} \leq 1$.

Remark 5.6 (Suboptimal order). The rate of convergence of order $1 / 2$ of Theorem 5.3 is due to the presence of $\int_{t^{n}}^{t^{n+1}} \sigma(t)\left\|\mathbf{u}_{t t}(t)\right\|_{0}^{2} d t$ in (5.12) and $\left\|\nabla p\left(t^{n+1}\right)\right\|_{0}$ in (5.13). To improve upon this, we must get rid of both terms.

This is precisely our next task. The main idea in obtaining an error estimate in $L^{2}\left(0, T ; \mathbf{L}^{2}(\Omega)\right)$ is to invert the main elliptic operator or, equivalently, multiply by a divergence free test function satisfying the Stokes equations. For $0 \leq n \leq N+1$, let $\left(\mathbf{v}^{n}, q^{n}\right) \in b f H_{0}^{1}(\Omega) \times L_{0}^{2}(\Omega)$ be the solution of

$$
\begin{aligned}
-\Delta \mathbf{v}^{n}+\nabla q^{n} & =\mathbf{E}^{n}, & & \text { in } \Omega, \\
\operatorname{div} \mathbf{v}^{n} & =0, & & \text { in } \Omega,
\end{aligned}
$$

with vanishing Dirichlet boundary condition $\mathbf{v}^{n}=\mathbf{0}$ on $\partial \Omega$. According to Assumption $\mathrm{A}\left[1\left(\mathbf{v}^{n}, q^{n}\right) \in b f H^{2}(\Omega) \times H^{1}(\Omega)\right.$ are strong solutions of (5.16) and satisfy

$$
\left\|\mathbf{v}^{n}\right\|_{2}+\left\|q^{n}\right\|_{1} \leq C\left\|\mathbf{E}^{n}\right\|_{0} .
$$

Theorem 5.7 (Full rate of convergence for velocity). If Assumptions A $1-A[$ hold, then the velocity error functions satisfy

$$
\left\|\mathbf{E}^{N+1}\right\|_{\mathbf{Z}^{*}}^{2}+\sum_{n=0}^{N}\left\|\mathbf{E}^{n+1}-\mathbf{E}^{n}\right\|_{\mathbf{Z}^{*}}^{2}+\mu \tau \sum_{n=0}^{N}\left(\left\|\mathbf{E}^{n+1}\right\|_{0}^{2}+\left\|\widehat{\mathbf{E}}^{n+1}\right\|_{0}^{2}\right) \leq C \tau^{2} .
$$


Proof. Let $\left(\mathbf{v}^{n}, q^{n}\right)$ be the solution of (5.16). Then, it satisfies

$$
\begin{aligned}
2\left\langle\mathbf{E}^{n+1}-\mathbf{E}^{n}, \mathbf{v}^{n+1}\right\rangle & =2\left\langle\nabla\left(\mathbf{v}^{n+1}-\mathbf{v}^{n}\right), \nabla \mathbf{v}^{n+1}\right\rangle \\
& =\left\|\nabla \mathbf{v}^{n+1}\right\|_{0}^{2}-\left\|\nabla \mathbf{v}^{n}\right\|_{0}^{2}+\left\|\nabla\left(\mathbf{v}^{n+1}-\mathbf{v}^{n}\right)\right\|_{0}^{2},
\end{aligned}
$$

as well as

$$
\begin{aligned}
2 \mu \tau\left\langle\nabla \widehat{\mathbf{E}}^{n+1}, \nabla \mathbf{v}^{n+1}\right\rangle & =2 \mu \tau\left\langle\widehat{\mathbf{E}}^{n+1}, \mathbf{E}^{n+1}-\nabla q^{n+1}\right\rangle \\
& =2 \mu \tau\left(\left\|\mathbf{E}^{n+1}\right\|_{0}^{2}-\left\langle\widehat{\mathbf{E}}^{n+1}, \nabla q^{n+1}\right\rangle\right),
\end{aligned}
$$

because of (5.3). Multiplying (5.9) by $2 \tau \mathbf{v}^{n+1} \in b f H_{0}^{1}(\Omega)$, thus yields

$$
\begin{aligned}
\left\|\nabla \mathbf{v}^{n+1}\right\|_{0}^{2}- & \left\|\nabla \mathbf{v}^{n}\right\|_{0}^{2}+\left\|\nabla\left(\mathbf{v}^{n+1}-\mathbf{v}^{n}\right)\right\|_{0}^{2}+2 \mu \tau\left\|\mathbf{E}^{n+1}\right\|_{0}^{2} \\
= & 2 \tau\left\langle\mathbf{R}^{n+1}, \mathbf{v}^{n+1}\right\rangle+2 \mu \tau\left\langle\widehat{\mathbf{E}}^{n+1}, \nabla q^{n+1}\right\rangle \\
& +2 \tau\left(\mathfrak{N}\left(\mathbf{u}^{n}, \widehat{\mathbf{u}}^{n+1}, \mathbf{v}^{n+1}\right)-\mathfrak{N}\left(\mathbf{u}\left(t^{n+1}\right), \mathbf{u}\left(t^{n+1}\right), \mathbf{v}^{n+1}\right)\right) \\
= & A_{1}+A_{2}+A_{3} .
\end{aligned}
$$

We now estimate $A_{1}$ to $A_{3}$ separately. Since $\mathbf{v}^{n+1} \in \mathbf{Z}$, defined in (3.2), we use (3.10) to find

$$
A_{1} \leq C \tau^{2} \int_{t^{n}}^{t^{n+1}}\left\|\mathbf{u}_{t t}\right\|_{\mathbf{Z}^{*}}^{2} d t+C \tau\left\|\nabla \mathbf{v}^{n+1}\right\|_{0}^{2} .
$$

To handle $A_{2}$, we first recall (5.2) and the orthogonality $\left\langle\mathbf{E}^{n+1}, \nabla q\right\rangle=0$ for all $q \in L^{2}(\Omega)$, because $\operatorname{div} \mathbf{E}^{n+1}=0$ and $\mathbf{E}^{n+1} \cdot \boldsymbol{\nu}=0$. Hence, (5.17) implies

$$
A_{2} \leq C \mu \tau\left\|\nabla\left(\phi^{n+1}-\phi^{n}\right)\right\|_{0}^{2}+\frac{\mu \tau}{2}\left\|\mathbf{E}^{n+1}\right\|_{0}^{2} .
$$

On the other hand, the convection term $A_{3}$ can be rewritten as follows:

$$
\begin{aligned}
A_{3}= & -2 \tau \mathfrak{N}\left(\mathbf{E}^{n}, \widehat{\mathbf{u}}^{n+1}, \mathbf{v}^{n+1}\right)-2 \tau \mathfrak{N}\left(\mathbf{u}\left(t^{n+1}\right)-\mathbf{u}\left(t^{n}\right), \widehat{\mathbf{u}}^{n+1}, \mathbf{v}^{n+1}\right) \\
& -2 \tau \mathfrak{N}\left(\mathbf{u}\left(t^{n+1}\right), \widehat{\mathbf{E}}^{n+1}, \mathbf{v}^{n+1}\right)=A_{3,1}+A_{3,2}+A_{3,3} .
\end{aligned}
$$

Since Theorem 5.3 and Assumption A1 yield $\left\|\mathbf{v}^{n+1}\right\|_{2} \leq C\left\|\mathbf{E}^{n+1}\right\|_{0} \leq C \tau^{1 / 2}$ and (3.5) gives $\left\|\mathbf{u}\left(t^{n+1}\right)\right\|_{2} \leq C$, appealing to (3.3), we easily deduce

$$
\begin{aligned}
A_{3,1} & =2 \tau \mathfrak{N}\left(\mathbf{E}^{n}, \widehat{\mathbf{E}}^{n+1}, \mathbf{v}^{n+1}\right)-2 \tau \mathfrak{N}\left(\mathbf{E}^{n}, \mathbf{u}\left(t^{n+1}\right), \mathbf{v}^{n+1}\right) \\
& \leq C \tau\left\|\mathbf{E}^{n}\right\|_{0}\left\|\nabla \widehat{\mathbf{E}}^{n+1}\right\|_{0}\left\|\mathbf{v}^{n+1}\right\|_{2}+C \tau\left\|\mathbf{E}^{n}\right\|_{0}\left\|\mathbf{u}\left(t^{n+1}\right)\right\|_{2}\left\|\nabla \mathbf{v}^{n+1}\right\|_{0} \\
& \leq \frac{\mu \tau}{4}\left\|\mathbf{E}^{n+1}\right\|_{0}^{2}+C \mu \tau\left\|\mathbf{E}^{n+1}-\mathbf{E}^{n}\right\|_{0}^{2}+\frac{C \tau^{2}}{\mu}\left\|\nabla \widehat{\mathbf{E}}^{n+1}\right\|_{0}^{2}+\frac{C \tau}{\mu}\left\|\nabla \mathbf{v}^{n+1}\right\|_{0}^{2} .
\end{aligned}
$$

Likewise, since $\left\|\mathbf{u}\left(t^{n+1}\right)-\mathbf{u}\left(t^{n}\right)\right\|_{0}^{2} \leq C \tau \int_{t^{n}}^{t^{n+1}}\left\|\mathbf{u}_{t}\right\|_{0}^{2} d t$, we have

$$
\begin{aligned}
A_{3,2} & =2 \tau \mathfrak{N}\left(\mathbf{u}\left(t^{n+1}\right)-\mathbf{u}\left(t^{n}\right), \widehat{\mathbf{E}}^{n+1}, \mathbf{v}^{n+1}\right)-2 \tau \mathfrak{N}\left(\mathbf{u}\left(t^{n+1}\right)-\mathbf{u}\left(t^{n}\right), \mathbf{u}\left(t^{n+1}\right), \mathbf{v}^{n+1}\right) \\
& \leq \frac{C \tau^{2}}{\mu}\left\|\nabla \widehat{\mathbf{E}}^{n+1}\right\|_{0}^{2}+\frac{C \tau}{\mu}\left\|\nabla \mathbf{v}^{n+1}\right\|_{0}^{2}+C \mu \tau^{2} \int_{t^{n}}^{t^{n+1}}\left\|\mathbf{u}_{t}(t)\right\|_{0}^{2} d t .
\end{aligned}
$$

Since $\operatorname{div} \mathbf{u}\left(t^{n+1}\right)=0$, we can exchange the last two arguments of $A_{3,3}$ to write

$$
A_{3,3} \leq C \tau\left\|\mathbf{u}\left(t^{n+1}\right)\right\|_{2}\left\|\widehat{\mathbf{E}}^{n+1}\right\|_{0}\left\|\nabla \mathbf{v}^{n+1}\right\|_{0} \leq \frac{\mu \tau}{4}\left\|\widehat{\mathbf{E}}^{n+1}\right\|_{0}^{2}+\frac{C \tau}{\mu}\left\|\nabla \mathbf{v}^{n+1}\right\|_{0}^{2} .
$$


Inserting $A_{3,1}-A_{3,3}$ into (5.22) and recalling (5.5) yield

$$
\begin{aligned}
A_{3} \leq & \frac{\mu \tau}{2}\left\|\mathbf{E}^{n+1}\right\|_{0}^{2}+C \mu \tau\left\|\mathbf{E}^{n+1}-\mathbf{E}^{n}\right\|_{0}^{2}+\frac{C \tau^{2}}{\mu}\left\|\nabla \widehat{\mathbf{E}}^{n+1}\right\|_{0}^{2} \\
& +\frac{\mu \tau}{2}\left\|\nabla\left(\phi^{n+1}-\phi^{n}\right)\right\|_{0}^{2}+\frac{C \tau}{\mu}\left\|\nabla \mathbf{v}^{n+1}\right\|_{0}^{2}+C \tau^{2} \int_{t^{n}}^{t^{n+1}}\left\|\mathbf{u}_{t}(t)\right\|_{0}^{2} d t .
\end{aligned}
$$

Combining (5.21) with $A_{1}-A_{3}$ and adding over $n$ from 0 to $N$ lead to

$$
\begin{aligned}
& \left\|\nabla \mathbf{v}^{N+1}\right\|_{0}^{2}+\sum_{n=0}^{N}\left\|\nabla\left(\mathbf{v}^{n+1}-\mathbf{v}^{n}\right)\right\|_{0}^{2}+\mu \tau \sum_{n=0}^{N}\left\|\mathbf{E}^{n+1}\right\|_{0}^{2} \\
& \leq \frac{C \tau}{\mu} \sum_{n=0}^{N}\left\|\nabla \mathbf{v}^{n+1}\right\|_{0}^{2}+C \mu \tau \sum_{n=0}^{N}\left(\left\|\nabla\left(\phi^{n+1}-\phi^{n}\right)\right\|_{0}^{2}+\left\|\mathbf{E}^{n+1}-\mathbf{E}^{n}\right\|_{0}^{2}\right) \\
& \quad+\frac{C \tau^{2}}{\mu} \sum_{n=0}^{N}\left\|\nabla \widehat{\mathbf{E}}^{n+1}\right\|_{0}^{2}+C \tau^{2} \int_{0}^{t^{N+1}}\left(\left\|\mathbf{u}_{t t}\right\|_{\mathbf{Z}^{*}}^{2}+\left\|\mathbf{u}_{t}(t)\right\|_{0}^{2}\right) d t .
\end{aligned}
$$

Making use of (5.7), in conjunction with (3.5) and (3.10), we arrive at

$$
\left\|\nabla \mathbf{v}^{N+1}\right\|_{0}^{2}+\sum_{n=0}^{N}\left\|\nabla\left(\mathbf{v}^{n+1}-\mathbf{v}^{n}\right)\right\|_{0}^{2}+\mu \tau \sum_{n=0}^{N}\left\|\mathbf{E}^{n+1}\right\|_{0}^{2} \leq C \tau^{2}+\frac{C \tau}{\mu} \sum_{n=0}^{N}\left\|\nabla \mathbf{v}^{n+1}\right\|_{0}^{2} .
$$

Applying the discrete Gronwall lemma allows us to remove the last term on the right-hand side. With the aid of Lemma 3.1, this implies (5.18) except for the estimate involving $\left\|\widehat{\mathbf{E}}^{n+1}\right\|_{0}^{2}$. The latter follows from (5.5) and (5.7) together and completes the proof.

We now embark on an error analysis for the time derivative of velocity. We use the notation

$$
\delta W^{n+1}:=\frac{W^{n+1}-W^{n}}{\tau}
$$

for any sequence $\left\{W^{n}\right\}_{n=0}^{N}$ and $\sigma^{n}:=\sigma\left(t^{n}\right)$ for $1 \leq n \leq N$. We first observe an immediate consequence of Theorem 5.7

Remark 5.8 (Estimate for $\left\|\nabla \delta \mathbf{v}^{1}\right\|_{0}$ ). Choosing $N=0$ in (5.23), we readily get

$$
\begin{aligned}
\left\|\nabla \mathbf{v}^{1}\right\|_{0}^{2} \leq & \frac{C \tau}{\mu}\left\|\nabla \mathbf{v}^{1}\right\|_{0}^{2}+C \mu \tau\left(\left\|\nabla \phi^{1}\right\|_{0}^{2}+\left\|\mathbf{E}^{1}\right\|_{0}^{2}\right) \\
& +\frac{C \tau^{2}}{\mu}\left\|\nabla \widehat{\mathbf{E}}^{1}\right\|_{0}^{2}+C \tau^{2} \int_{0}^{t^{1}}\left(\left\|\mathbf{u}_{t t}\right\|_{\mathbf{Z}^{*}}^{2}+\left\|\mathbf{u}_{t}(t)\right\|_{0}^{2}\right) d t \\
\leq & C \tau^{3}+C \tau^{2} \int_{0}^{t^{1}}\left(\sigma(t)\left\|\mathbf{u}_{t t}\right\|_{0}^{2}+\left\|\mathbf{u}_{t t}\right\|_{\mathbf{Z}^{*}}^{2}+\left\|\mathbf{u}_{t}(t)\right\|_{0}^{2}\right) d t \leq C \tau^{2},
\end{aligned}
$$

with the aid of Remark [5.5 and Theorem [5.7. If Assumption A4 is also valid, then Remark [5.5leads to a right-hand side $\leq C \tau^{3}$. Since $\mathbf{v}^{0}=\mathbf{0}$, this gives $\left\|\nabla \delta \mathbf{v}^{1}\right\|_{0} \leq C$ provided Assumptions $\mathrm{A}\left[\mathrm{H}-\mathrm{A}\right.$ [3hold and $\left\|\nabla \delta \mathbf{v}^{1}\right\|_{0} \leq C \tau^{1 / 2}$ provided Assumption A4 is valid as well. 
Lemma 5.9 (Enhanced stability). If Assumptions A1-A hold, then the error functions satisfy the weighted a priori bounds

$$
\begin{array}{r}
\sum_{n=1}^{N} \sigma^{n+1}\left(\left\|\delta \mathbf{E}^{n+1}-\delta \mathbf{E}^{n}\right\|_{0}^{2}+\left\|\nabla\left(\delta \phi^{n+1}-\delta \phi^{n}\right)\right\|_{0}^{2}+\mu \tau\left\|\nabla \delta \widehat{\mathbf{E}}^{n+1}\right\|_{0}^{2}\right) \\
+\sigma^{N+1}\left\|\delta \mathbf{E}^{N+1}\right\|_{0}^{2}+\mu \sigma^{N+1} \tau\left\|\Delta \delta \phi^{N+1}\right\|_{0}^{2} \leq C .
\end{array}
$$

If Assumption A母 is also valid, then (5.24) becomes uniform, namely without weights.

Since we intend to derive an $L^{2}$-based estimate, the main difficulty is to deal with pressure. We postpone the proof of Lemma 5.9 and instead apply it to derive the desired error estimate.

Theorem 5.10 (Error estimate for time-derivative of velocity). If Assumptions A1-A 3 hold, then the error functions satisfy the weighted estimates

$$
\sigma^{N+1}\left\|\delta \mathbf{E}^{N+1}\right\|_{\mathbf{Z}^{*}}^{2}+\sum_{n=1}^{N} \sigma^{n+1}\left\|\delta \mathbf{E}^{n+1}-\delta \mathbf{E}^{n}\right\|_{\mathbf{Z}^{*}}^{2}+\mu \tau \sum_{n=1}^{N} \sigma^{n+1}\left\|\delta \mathbf{E}^{n+1}\right\|_{0}^{2} \leq C \tau .
$$

If Assumption A4 is also valid, then the following uniform error estimates hold:

$$
\left\|\delta \mathbf{E}^{N+1}\right\|_{\mathbf{Z}^{*}}^{2}+\sum_{n=1}^{N}\left\|\delta \mathbf{E}^{n+1}-\delta \mathbf{E}^{n}\right\|_{\mathbf{Z}^{*}}^{2}+\mu \tau \sum_{n=1}^{N}\left\|\delta \mathbf{E}^{n+1}\right\|_{0}^{2} \leq C \tau .
$$

Proof. Let $\left(\mathbf{v}^{n}, q^{n}\right) \in b f H_{0}^{1}(\Omega) \times L_{0}^{2}(\Omega)$ be the solution of (5.16); then

$$
\left\|\delta \mathbf{v}^{n+1}\right\|_{2}+\left\|\delta q^{n+1}\right\|_{1} \leq C\left\|\delta \mathbf{E}^{n+1}\right\|_{0} .
$$

Subtracting two consecutive expressions (5.9), multiplying by $2 \delta \mathbf{v}^{n+1} \in b f H_{0}^{1}(\Omega)$, and recalling the argument leading to (5.19) and (5.20), yield

$$
\begin{aligned}
&\left\|\nabla \delta \mathbf{v}^{n+1}\right\|_{0}^{2}-\left\|\nabla \delta \mathbf{v}^{n}\right\|_{0}^{2}+\left\|\nabla\left(\delta \mathbf{v}^{n+1}-\delta \mathbf{v}^{n}\right)\right\|_{0}^{2}+2 \mu \tau\left\|\delta \mathbf{E}^{n+1}\right\|_{0}^{2} \\
&= 2 \mu \tau\left\langle\nabla \delta q^{n+1}, \nabla \delta\left(\phi^{n+1}-\phi^{n}\right)\right\rangle+2\left\langle\mathbf{R}^{n+1}-\mathbf{R}^{n}, \delta \mathbf{v}^{n+1}\right\rangle \\
&-2\left(\mathfrak{N}\left(\mathbf{u}\left(t^{n+1}\right), \mathbf{u}\left(t^{n+1}\right), \delta \mathbf{v}^{n+1}\right)-\mathfrak{N}\left(\mathbf{u}\left(t^{n}\right), \mathbf{u}\left(t^{n}\right), \delta \mathbf{v}^{n+1}\right)\right. \\
&\left.-\mathfrak{N}\left(\mathbf{u}^{n}, \widehat{\mathbf{u}}^{n+1}, \delta \mathbf{v}^{n+1}\right)+\mathfrak{N}\left(\mathbf{u}^{n-1}, \widehat{\mathbf{u}}^{n}, \delta \mathbf{v}^{n+1}\right)\right)=A_{1}+A_{2}+A_{3} .
\end{aligned}
$$

We now estimate each term $A_{i}$ separately. We first use (5.25) to write

$$
A_{1} \leq \frac{\mu \tau}{5}\left\|\delta \mathbf{E}^{n+1}\right\|_{0}^{2}+C \mu \tau\left\|\nabla\left(\delta \phi^{n+1}-\delta \phi^{n}\right)\right\|_{0}^{2} .
$$

Since $\mathbf{v}^{n+1} \in \mathbf{Z}$, space defined in (3.2), we use (3.10) to find

$$
\begin{aligned}
A_{2}= & 2\left\langle\mathbf{R}^{n+1}, \delta \mathbf{v}^{n+1}\right\rangle-2\left\langle\mathbf{R}^{n}, \delta \mathbf{v}^{n}\right\rangle-2\left\langle\mathbf{R}^{n}, \delta \mathbf{v}^{n+1}-\delta \mathbf{v}^{n}\right\rangle \\
\leq & 2\left\langle\mathbf{R}^{n+1}, \delta \mathbf{v}^{n+1}\right\rangle-2\left\langle\mathbf{R}^{n}, \delta \mathbf{v}^{n}\right\rangle \\
& +C \tau \int_{t^{n-1}}^{t^{n}}\left\|\mathbf{u}_{t t}(t)\right\|_{\mathbf{Z}^{*}}^{2} d t+\frac{1}{2}\left\|\nabla\left(\delta \mathbf{v}^{n+1}-\delta \mathbf{v}^{n}\right)\right\|_{0}^{2} .
\end{aligned}
$$


On the other hand, an elementary manipulation of $A_{3}$ gives

$$
\begin{aligned}
A_{3}= & -2 \mathfrak{N}\left(\mathbf{u}\left(t^{n+1}\right)-2 \mathbf{u}\left(t^{n}\right)+\mathbf{u}\left(t^{n-1}\right), \mathbf{u}\left(t^{n+1}\right), \delta \mathbf{v}^{n+1}\right) \\
& -2 \mathfrak{N}\left(\mathbf{E}^{n}-\mathbf{E}^{n-1}, \mathbf{u}\left(t^{n+1}\right), \delta \mathbf{v}^{n+1}\right)-2 \mathfrak{N}\left(\mathbf{u}^{n}-\mathbf{u}^{n-1}, \widehat{\mathbf{E}}^{n+1}, \delta \mathbf{v}^{n+1}\right) \\
& -2 \mathfrak{N}\left(\mathbf{u}\left(t^{n}\right)-\mathbf{u}\left(t^{n-1}\right), \mathbf{u}\left(t^{n+1}\right)-\mathbf{u}\left(t^{n}\right), \delta \mathbf{v}^{n+1}\right) \\
& -2 \mathfrak{N}\left(\mathbf{E}^{n-1}, \mathbf{u}\left(t^{n+1}\right)-\mathbf{u}\left(t^{n}\right), \delta \mathbf{v}^{n+1}\right)-2 \mathfrak{N}\left(\mathbf{u}^{n-1}, \widehat{\mathbf{E}}^{n+1}-\widehat{\mathbf{E}}^{n}, \delta \mathbf{v}^{n+1}\right) \\
= & A_{3,1}+A_{3,2}+A_{3,3}+A_{3,4}+A_{3,5}+A_{3,6} .
\end{aligned}
$$

To bound $A_{3,1}$, we first observe

$$
\mathbf{u}\left(t^{n+1}\right)-2 \mathbf{u}\left(t^{n}\right)+\mathbf{u}\left(t^{n-1}\right)=\int_{t^{n-1}}^{t^{n}}\left(t-t^{n-1}\right) \mathbf{u}_{t t}(t) d t+\int_{t^{n}}^{t^{n+1}}\left(t^{n+1}-t\right) \mathbf{u}_{t t}(t) d t
$$

whence

$$
\left\|\mathbf{u}\left(t^{n+1}\right)-2 \mathbf{u}\left(t^{n}\right)+\mathbf{u}\left(t^{n-1}\right)\right\|_{0}^{2} \leq C \tau^{2} \int_{t^{n-1}}^{t^{n+1}} \sigma(t)\left\|\mathbf{u}_{t t}(t)\right\|_{0}^{2} d t .
$$

This, together with $\left\|\mathbf{u}\left(t^{n+1}\right)\right\|_{2} \leq M$ (see (3.5)), and the aid of (3.3), yield

$$
\begin{aligned}
A_{3,1} & \leq C\left\|\mathbf{u}\left(t^{n+1}\right)-2 \mathbf{u}\left(t^{n}\right)+\mathbf{u}\left(t^{n-1}\right)\right\|_{0}\left\|\mathbf{u}\left(t^{n+1}\right)\right\|_{2}\left\|\nabla \delta \mathbf{v}^{n+1}\right\|_{0} \\
& \leq C \tau \int_{t^{n-1}}^{t^{n+1}} \sigma(t)\left\|\mathbf{u}_{t t}(t)\right\|_{0}^{2} d t+C \tau\left\|\nabla \delta \mathbf{v}^{n+1}\right\|_{0}^{2}
\end{aligned}
$$

and

$$
\begin{aligned}
A_{3,2} & \leq C \tau\left\|\delta \mathbf{E}^{n}\right\|_{0}\left\|\mathbf{u}\left(t^{n+1}\right)\right\|_{2}\left\|\nabla \delta \mathbf{v}^{n+1}\right\|_{0} \\
& \leq \frac{\mu \tau}{5}\left(\left\|\delta \mathbf{E}^{n+1}\right\|_{0}^{2}+\left\|\delta \mathbf{E}^{n+1}-\delta \mathbf{E}^{n}\right\|_{0}^{2}\right)+\frac{C \tau}{\mu}\left\|\nabla \delta \mathbf{v}^{n+1}\right\|_{0}^{2} .
\end{aligned}
$$

To estimate $A_{3,3}-A_{3,6}$, we use to the following estimates proved in Lemma 5.9 and Theorem 5.3 .

$$
\sigma^{n}\left\|\mathbf{E}^{n}-\mathbf{E}^{n-1}\right\|_{0}^{2} \leq C \tau^{2}, \quad\left\|\mathbf{E}^{n+1}\right\|_{0}^{2}+\left\|\widehat{\mathbf{E}}^{n+1}\right\|_{0}^{2} \leq C \tau .
$$

Since

$$
\left\|\mathbf{u}\left(t^{n}\right)-\mathbf{u}\left(t^{n-1}\right)\right\|_{1}^{2} \leq \tau \int_{t^{n-1}}^{t^{n}}\left\|\mathbf{u}_{t}(t)\right\|_{1}^{2} d t \leq C \tau
$$

thanks to Lemma 3.4 we apply (15.25) in conjunction with (3.3) to deduce

$$
\begin{aligned}
A_{3,3} & =2 \mathfrak{N}\left(\left(\mathbf{E}^{n}-\mathbf{E}^{n-1}\right)-\left(\mathbf{u}\left(t^{n}\right)-\mathbf{u}\left(t^{n-1}\right)\right), \widehat{\mathbf{E}}^{n+1}, \delta \mathbf{v}^{n+1}\right) \\
& \leq C\left(\left\|\mathbf{E}^{n}-\mathbf{E}^{n-1}\right\|_{0}\left\|\widehat{\mathbf{E}}^{n+1}\right\|_{1}+\left\|\mathbf{u}\left(t^{n}\right)-\mathbf{u}\left(t^{n-1}\right)\right\|_{1}\left\|\widehat{\mathbf{E}}^{n+1}\right\|_{0}\right)\left\|\delta \mathbf{v}^{n+1}\right\|_{2} \\
& \leq \frac{C \tau}{\mu}\left(\frac{1}{\sigma^{n}}\left\|\nabla \widehat{\mathbf{E}}^{n+1}\right\|_{0}^{2}+\int_{t^{n-1}}^{t^{n}}\left\|\mathbf{u}_{t}(t)\right\|_{1}^{2} d t\right)+\frac{\mu \tau}{5}\left\|\delta \mathbf{E}^{n+1}\right\|_{0}^{2} .
\end{aligned}
$$

In view of (5.29), we readily obtain

$$
\begin{aligned}
A_{3,4} & \leq C\left\|\mathbf{u}\left(t^{n}\right)-\mathbf{u}\left(t^{n-1}\right)\right\|_{1}\left\|\mathbf{u}\left(t^{n+1}\right)-\mathbf{u}\left(t^{n}\right)\right\|_{1}\left\|\delta \mathbf{v}^{n+1}\right\|_{1} \\
& \leq C \tau \int_{t^{n}}^{t^{n+1}}\left\|\mathbf{u}_{t}(t)\right\|_{1}^{2} d t+C \tau\left\|\nabla \delta \mathbf{v}^{n+1}\right\|_{0}^{2} .
\end{aligned}
$$


With the aid of (3.3), (5.28) and (5.29), we can bound $A_{3,5}$ and $A_{3,6}$ as follows:

$$
A_{3,5} \leq C \tau\left\|\mathbf{E}^{n-1}\right\|_{0}\left\|\delta \mathbf{u}\left(t^{n+1}\right)\right\|_{1}\left\|\delta \mathbf{v}^{n+1}\right\|_{2} \leq \frac{C \tau}{\mu} \int_{t^{n}}^{t^{n+1}}\left\|\mathbf{u}_{t}(t)\right\|_{1}^{2} d t+\frac{\mu \tau}{5}\left\|\delta \mathbf{E}^{n+1}\right\|_{0}^{2}
$$

and

$$
\begin{aligned}
& A_{3,6}=2 \tau \mathfrak{N}\left(\mathbf{E}^{n-1}-\mathbf{u}\left(t^{n-1}\right), \delta \widehat{\mathbf{E}}^{n+1}, \delta \mathbf{v}^{n+1}\right) \\
& \leq C \tau\left(\left\|\mathbf{E}^{n-1}\right\|_{0}\left\|\delta \widehat{\mathbf{E}}^{n+1}\right\|_{1}\left\|\delta \mathbf{v}^{n+1}\right\|_{2}+\left\|\mathbf{u}\left(t^{n-1}\right)\right\|_{2}\left\|\delta \widehat{\mathbf{E}}^{n+1}\right\|_{0}\left\|\delta \mathbf{v}^{n+1}\right\|_{1}\right) \\
& \leq \frac{C \tau^{2}}{\mu}\left\|\nabla \delta \widehat{\mathbf{E}}^{n+1}\right\|_{0}^{2}+\frac{C \tau}{\mu}\left\|\nabla \delta \mathbf{v}^{n+1}\right\|_{0}^{2}+\frac{\mu \tau}{5}\left\|\delta \mathbf{E}^{n+1}\right\|_{0}^{2}+C \tau\left\|\nabla\left(\delta \phi^{n+1}-\delta \phi^{n}\right)\right\|_{0}^{2} .
\end{aligned}
$$

We now multiply both sides of $(5.26)$ by the weight $\sigma^{n+1}$ and sum over $n$ for $1 \leq n \leq N$. We first examine the first two terms on the left-hand side of (5.26), which can be rewritten as follows:

$$
\begin{aligned}
& \sum_{n=1}^{N}\left(\sigma^{n+1}\left\|\nabla \delta \mathbf{v}^{n+1}\right\|_{0}^{2}-\sigma^{n}\left\|\nabla \delta \mathbf{v}^{n}\right\|_{0}^{2}-\left(\sigma^{n+1}-\sigma^{n}\right)\left\|\nabla \delta \mathbf{v}^{n}\right\|_{0}^{2}\right) \\
& \geq \sigma^{N+1}\left\|\nabla \delta \mathbf{v}^{N+1}\right\|_{0}^{2}-\sigma^{1}\left\|\nabla \delta \mathbf{v}^{1}\right\|_{0}^{2}-\tau \sum_{n=1}^{N}\left\|\nabla \delta \mathbf{v}^{n}\right\|_{0}^{2} \geq \sigma^{N+1}\left\|\nabla \delta \mathbf{v}^{N+1}\right\|_{0}^{2}-C \tau .
\end{aligned}
$$

This is indeed a consequence of $\mathbf{E}^{0}=\mathbf{v}^{0}=\mathbf{0}, \sigma^{1}=\tau$, and Remark 5.8 which give $\sigma^{1}\left\|\nabla \delta \mathbf{v}^{1}\right\|_{0}^{2} \leq C \tau$, as well as Lemma 3.1 and Theorem 5.7, which imply $\sum_{n=1}^{N}\left\|\nabla \delta \mathbf{v}^{n}\right\|_{0}^{2} \leq C$. Similarly, we analyze the first two terms on the right-hand side of (5.27), which become

$$
\begin{aligned}
& 2 \sum_{n=1}^{N}\left(\sigma^{n+1}\left\langle\mathbf{R}^{n+1}, \delta \mathbf{v}^{n+1}\right\rangle-\sigma^{n}\left\langle\mathbf{R}^{n}, \delta \mathbf{v}^{n}\right\rangle-\left(\sigma^{n+1}-\sigma^{n}\right)\left\langle\mathbf{R}^{n}, \delta \mathbf{v}^{n}\right\rangle\right) \\
& \leq 2 \sigma^{N+1}\left\|\mathbf{R}^{N+1}\right\|_{\mathbf{Z}^{*}}\left\|\delta \mathbf{v}^{N+1}\right\|_{1}+2 \tau\left\|\mathbf{R}^{1}\right\|_{\mathbf{Z}^{*}}\left\|\delta \mathbf{v}^{1}\right\|_{1}+2 \tau \sum_{n=1}^{N}\left\|\mathbf{R}^{n}\right\|_{\mathbf{Z}^{*}}\left\|\delta \mathbf{v}^{n}\right\|_{1} \\
& \leq \frac{\sigma^{N+1}}{2}\left\|\nabla \delta \mathbf{v}^{N+1}\right\|_{0}^{2}+C \tau+C \tau \int_{0}^{t^{N+1}}\left\|\mathbf{u}_{t t}(t)\right\|_{\mathbf{Z}^{*}}^{2} d t .
\end{aligned}
$$

Inserting the above estimates into (5.26) gives

$$
\begin{aligned}
& \frac{\sigma^{N+1}}{2}\left\|\nabla \delta \mathbf{v}^{N+1}\right\|_{0}^{2}+\sum_{n=1}^{N} \sigma^{n+1}\left(\frac{1}{2}\left\|\nabla\left(\delta \mathbf{v}^{n+1}-\delta \mathbf{v}^{n}\right)\right\|_{0}^{2}+\mu \tau\left\|\delta \mathbf{E}^{n+1}\right\|_{0}^{2}\right) \\
& \leq C \tau+C \tau \int_{0}^{t^{N+1}}\left(\left\|\mathbf{u}_{t t}\right\|_{\mathbf{Z}^{*}}^{2}+\sigma(t)\left\|\mathbf{u}_{t t}\right\|_{0}^{2}+\left\|\mathbf{u}_{t}\right\|_{1}^{2}\right) d t \\
& \quad+C \tau \sum_{n=1}^{N} \sigma^{n+1}\left(\left\|\nabla \delta \mathbf{v}^{n+1}\right\|_{0}^{2}+\tau\left\|\nabla \delta \widehat{\mathbf{E}}^{n+1}\right\|_{0}^{2}+\left\|\delta \mathbf{E}^{n+1}-\delta \mathbf{E}^{n}\right\|_{0}^{2}\right) \\
& \quad+C \tau \sum_{n=1}^{N} \sigma^{n+1}\left\|\nabla\left(\delta \phi^{n+1}-\delta \phi^{n}\right)\right\|_{0}^{2}+C \tau \sum_{n=1}^{N} \frac{\sigma^{n+1}}{\sigma^{n}}\left\|\nabla \widehat{\mathbf{E}}^{n+1}\right\|_{0}^{2} .
\end{aligned}
$$

Since $\frac{\sigma^{n+1}}{\sigma^{n}} \leq 2$ for $n \geq 1$, recalling Lemmas [3.4, 3.6] and [5.9] and Theorems [5.3] and 5.7, and using the discrete Gronwall lemma, the asserted weighted estimate follows. 
To derive the uniform error estimate, we do not multiply (5.26) by $\sigma^{n+1}$. Since now $\left\|\nabla \delta \mathbf{v}^{1}\right\|_{0}^{2} \leq C \tau$, according to Remark [5.8 we immediately see that

$$
\sum_{n=1}^{N}\left\|\nabla \delta \mathbf{v}^{n+1}\right\|_{0}^{2}-\left\|\nabla \delta \mathbf{v}^{n}\right\|_{0}^{2} \geq-C \tau+\left\|\nabla \delta \mathbf{v}^{N+1}\right\|_{0}^{2}
$$

Likewise, using (3.11), we easily arrive at

$$
2 \sum_{n=1}^{N}\left(\left\langle\mathbf{R}^{n+1}, \delta \mathbf{v}^{n+1}\right\rangle-\left\langle\mathbf{R}^{n}, \delta \mathbf{v}^{n}\right\rangle\right) \leq C \tau^{3 / 2}+\frac{1}{2}\left\|\nabla \mathbf{v}^{n+1}\right\|_{0}^{2} .
$$

Since we can remove $\sigma^{n}$ in (5.28), and thus in the bound for $A_{3,3}$, we end up with an expression similar to (5.30) but without weights. Proceeding as before, we conclude the desired estimate via the discrete Gronwall lemma.

Remark 5.11 (Optimality). The above error estimate $\tau \sum_{n=0}^{N} \sigma^{n+1}\left\|\delta \mathbf{E}^{n+1}\right\|_{0}^{2} \leq C \tau$ is consistent with the regularity $\int_{0}^{T} \sigma\left\|\mathbf{u}_{t t}\right\|_{0}^{2} d t$ of Lemma 3.4 In fact, the loss of half an order is customary unless the PDE corresponds to an angle-bounded operator [16. This is not the case of (1.1).

Proof of Lemma 5.9. Subtracting two consecutive formulas (5.9) and multiplying by $2 \delta \widehat{\mathbf{E}}^{n+1}=2 \delta \mathbf{E}^{n+1}+\nabla\left(\delta \phi^{n+1}-\delta \phi^{n}\right) \in b f H_{0}^{1}(\Omega)$ yield

$$
\begin{aligned}
& \left\|\delta \mathbf{E}^{n+1}\right\|_{0}^{2}-\left\|\delta \mathbf{E}^{n}\right\|_{0}^{2}+\left\|\delta \mathbf{E}^{n+1}-\delta \mathbf{E}^{n}\right\|_{0}^{2}+2\left\|\nabla\left(\delta \phi^{n+1}-\delta \phi^{n}\right)\right\|_{0}^{2} \\
& +2 \mu \tau\left\|\nabla \delta \widehat{\mathbf{E}}^{n+1}\right\|_{0}^{2}=2\left\langle\mathbf{R}^{n+1}-\mathbf{R}^{n}, \delta \widehat{\mathbf{E}}^{n+1}\right\rangle-2 \mu \tau\left\langle\Delta \delta \phi^{n}, \operatorname{div} \delta \widehat{\mathbf{E}}^{n+1}\right\rangle \\
& +2\left\langle p\left(t^{n+1}\right)-p\left(t^{n}\right), \operatorname{div} \delta \widehat{\mathbf{E}}^{n+1}\right\rangle-2\left(\mathfrak{N}\left(\mathbf{u}\left(t^{n+1}\right), \mathbf{u}\left(t^{n+1}\right), \delta \widehat{\mathbf{E}}^{n+1}\right)\right. \\
& \left.-\mathfrak{N}\left(\mathbf{u}^{n}, \widehat{\mathbf{u}}^{n+1}, \delta \widehat{\mathbf{E}}^{n+1}\right)-\mathfrak{N}\left(\mathbf{u}\left(t^{n}\right), \mathbf{u}\left(t^{n}\right), \delta \widehat{\mathbf{E}}^{n+1}\right)+\mathfrak{N}\left(\mathbf{u}^{n-1}, \widehat{\mathbf{u}}^{n}, \delta \widehat{\mathbf{E}}^{n+1}\right)\right) \\
& =A_{1}+A_{2}+A_{3}+A_{4} .
\end{aligned}
$$

We now estimate each term $A_{1}$ to $A_{4}$ separately. We easily find out that

$$
A_{1} \leq 2\left\|\mathbf{R}^{n+1}-\mathbf{R}^{n}\right\|_{\mathbf{Z}^{*}}\left\|\delta \widehat{\mathbf{E}}^{n+1}\right\|_{1} \leq \frac{\mu \tau}{8}\left\|\nabla \delta \widehat{\mathbf{E}}^{n+1}\right\|_{0}^{2}+\frac{C}{\mu} \int_{t^{n}}^{t^{n+1}}\left\|\mathbf{u}_{t t}(t)\right\|_{\mathbf{Z}^{*}}^{2} d t,
$$

as well as

$$
A_{3} \leq 2\left\|p\left(t^{n+1}\right)-p\left(t^{n}\right)\right\|_{0}\left\|\nabla \delta \widehat{\mathbf{E}}^{n+1}\right\|_{0} \leq \frac{\mu \tau}{8}\left\|\nabla \delta \widehat{\mathbf{E}}^{n+1}\right\|_{0}^{2}+\frac{C}{\mu} \int_{t^{n}}^{t^{n+1}}\left\|p_{t}\right\|_{0}^{2} d t,
$$

the latter being a consequence of (3.6). Making use of (5.2) and (5.4), we arrive at

$$
\begin{aligned}
A_{2} & =-2 \mu \tau\left\langle\Delta \delta \phi^{n}, \Delta\left(\delta \phi^{n+1}-\delta \phi^{n}\right)\right\rangle \\
& \leq-\mu \tau\left(\left\|\Delta \delta \phi^{n+1}\right\|_{0}^{2}-\left\|\Delta \delta \phi^{n}\right\|_{0}^{2}\right)+\mu \tau\left\|\nabla \delta \widehat{\mathbf{E}}^{n+1}\right\|_{0}^{2} .
\end{aligned}
$$

On the other hand, the convection term $A_{4}$ can be rewritten as follows:

$$
\begin{aligned}
A_{4}= & -2\left(\mathfrak{N}\left(\mathbf{u}\left(t^{n+1}\right)-\mathbf{u}\left(t^{n}\right), \mathbf{u}\left(t^{n+1}\right), \delta \widehat{\mathbf{E}}^{n+1}\right)+\mathfrak{N}\left(\mathbf{E}^{n}, \mathbf{u}\left(t^{n+1}\right), \delta \widehat{\mathbf{E}}^{n+1}\right)\right. \\
& \left.-\mathfrak{N}\left(\mathbf{u}\left(t^{n}\right)-\mathbf{u}\left(t^{n-1}\right), \mathbf{u}\left(t^{n}\right), \delta \widehat{\mathbf{E}}^{n+1}\right)-\mathfrak{N}\left(\mathbf{E}^{n-1}, \mathbf{u}\left(t^{n}\right), \delta \widehat{\mathbf{E}}^{n+1}\right)\right) \\
& -2\left(\mathfrak{N}\left(\mathbf{u}^{n}, \widehat{\mathbf{E}}^{n+1}, \delta \widehat{\mathbf{E}}^{n+1}\right)-\mathfrak{N}\left(\mathbf{u}^{n-1}, \widehat{\mathbf{E}}^{n}, \delta \widehat{\mathbf{E}}^{n+1}\right)\right)=A_{4,1}+A_{4,2} .
\end{aligned}
$$


We now recall that $\left\|\mathbf{u}\left(t^{n}\right)\right\|_{2} \leq C$ (see (3.5.5) ) and use (3.3) to arrive at

$$
A_{4,1} \leq \frac{\mu \tau}{8}\left\|\nabla \delta \widehat{\mathbf{E}}^{n+1}\right\|_{0}^{2}+\frac{C}{\mu \tau}\left(\left\|\mathbf{E}^{n}\right\|_{0}^{2}+\left\|\mathbf{E}^{n-1}\right\|_{0}^{2}\right)+\frac{C}{\mu} \int_{t^{n-1}}^{t^{n+1}}\left\|\mathbf{u}_{t}(t)\right\|_{0}^{2} d t
$$

We first rewrite $A_{4,2}$ as follows invoking the crucial properties of $\mathfrak{N}$ of Lemma 3.2

$$
\begin{aligned}
A_{4,2} & =\frac{2}{\tau} \mathfrak{N}\left(\mathbf{u}^{n}-\mathbf{u}^{n-1}, \widehat{\mathbf{E}}^{n+1}, \widehat{\mathbf{E}}^{n}\right) \\
& =2 \mathfrak{N}\left(\mathbf{u}\left(t^{n}\right)-\mathbf{u}\left(t^{n-1}\right), \delta \widehat{\mathbf{E}}^{n+1}, \widehat{\mathbf{E}}^{n}\right)-2 \mathfrak{N}\left(\mathbf{E}^{n}-\mathbf{E}^{n-1}, \delta \widehat{\mathbf{E}}^{n+1}, \widehat{\mathbf{E}}^{n}\right)=B_{1}^{n}+B_{2}^{n} .
\end{aligned}
$$

Since Theorem 5.3 yields $\left\|\widehat{\mathbf{E}}^{n}\right\|_{1} \leq C$, we obtain

$$
\begin{aligned}
B_{1}^{n} & \leq C\left\|\mathbf{u}\left(t^{n}\right)-\mathbf{u}\left(t^{n-1}\right)\right\|_{1}\left\|\delta \widehat{\mathbf{E}}^{n+1}\right\|_{1}\left\|\widehat{\mathbf{E}}^{n}\right\|_{1} \\
& \leq \frac{\mu \tau}{8}\left\|\nabla \delta \widehat{\mathbf{E}}^{n+1}\right\|_{0}+\frac{C}{\mu} \int_{t^{n-1}}^{t^{n}}\left\|\mathbf{u}_{t}(t)\right\|_{1}^{2} d t .
\end{aligned}
$$

Instead of estimating $B_{2}^{n}$, we first insert the above estimates into (5.31), multiply by the weight $\sigma^{n+1}$, and add over $n$ from 1 to $N$. Arguing as in Theorem 5.10. namely using Theorem5.3 and Remark 5.5, the first two terms in (5.31) become

$$
\sigma^{N+1}\left\|\delta \mathbf{E}^{N+1}\right\|_{0}^{2}-\sigma^{1}\left\|\delta \mathbf{E}^{1}\right\|_{0}^{2}-\tau \sum_{n=1}^{N}\left\|\delta \mathbf{E}^{n}\right\|_{0}^{2} \geq-C+\sigma^{N+1}\left\|\delta \mathbf{E}^{N+1}\right\|_{0}^{2}
$$

On the other hand, we resort to the property $\frac{\sigma^{n+1}}{\sigma^{n}} \leq 2$ for $n \geq 1$ to write

$$
\sum_{n=1}^{N} \sigma^{n+1} A_{3} \leq \frac{\mu \tau}{8} \sum_{n=1}^{N} \sigma^{n+1}\left\|\nabla \delta \widehat{\mathbf{E}}^{n+1}\right\|_{0}^{2}+\frac{C}{\mu} \int_{t^{1}}^{t^{N+1}} \sigma(t)\left\|p_{t}\right\|_{0}^{2} d t .
$$

Collecting all these estimates and using Lemma 3.4 we obtain

$$
\begin{aligned}
& \sigma^{N+1}\left\|\delta \mathbf{E}^{N+1}\right\|_{0}^{2}+\sum_{n=1}^{N} \sigma^{n+1}\left\|\delta \mathbf{E}^{n+1}-\delta \mathbf{E}^{n}\right\|_{0}^{2}+\frac{\mu \tau}{2} \sum_{n=1}^{N} \sigma^{n+1}\left\|\nabla \delta \widehat{\mathbf{E}}^{n+1}\right\|_{0}^{2} \\
& +\mu \tau \sigma^{N+1}\left\|\Delta \delta \phi^{N+1}\right\|_{0}^{2}+2 \sum_{n=1}^{N} \sigma^{n+1}\left\|\nabla \delta\left(\phi^{n+1}-\phi^{n}\right)\right\|_{0}^{2} \leq C+\sum_{n=1}^{N} \sigma^{n+1} B_{2}^{n} .
\end{aligned}
$$

We now deal with $B_{2}^{n}$ in two steps via (3.4). We first use $\left\|\mathbf{E}^{n}-\mathbf{E}^{n-1}\right\|_{0} \leq C \tau^{\frac{1}{2}}$ and $\left\|\mathbf{E}^{n}\right\|_{1} \leq\left\|\widehat{\mathbf{E}}^{n}\right\|_{1} \leq C$, which results from Theorem 5.3 and (5.6), to deduce

$$
B_{2}^{n} \leq C\left\|\mathbf{E}^{n}-\mathbf{E}^{n-1}\right\|_{0}^{\frac{1}{2}}\left\|\mathbf{E}^{n}-\mathbf{E}^{n-1}\right\|_{1}^{\frac{1}{2}}\left\|\delta \widehat{\mathbf{E}}^{n+1}\right\|_{1}\left\|\widehat{\mathbf{E}}^{n}\right\|_{1},
$$

whence

$$
\sum_{n=1}^{N} \sigma^{n+1} B_{2}^{n} \leq \frac{\mu \tau}{4} \sum_{n=1}^{N} \sigma^{n+1}\left\|\nabla \delta \widehat{\mathbf{E}}^{n+1}\right\|_{0}^{2}+\frac{C}{\mu \tau^{1 / 2}} \sum_{n=1}^{N}\left\|\nabla \widehat{\mathbf{E}}^{n}\right\|_{0}^{2} .
$$

Since $\sum_{n=1}^{N}\left\|\nabla \widehat{\mathbf{E}}^{n}\right\|_{0}^{2} \leq C$ according to Theorem [5.3, (5.34) gives the improvement

$$
\sigma^{n+1}\left\|\mathbf{E}^{n+1}-\mathbf{E}^{n}\right\|_{0}^{2} \leq C \tau^{\frac{3}{2}}, \quad \sigma^{n+1}\left\|\nabla\left(\mathbf{E}^{n+1}-\mathbf{E}^{n}\right)\right\|_{0}^{2} \leq C \tau^{\frac{1}{2}}
$$

over the starting condition for the 1 st step. Using this to estimate $B_{2}^{n}$ again, we discover that

$$
B_{2}^{n} \leq \frac{\mu \tau}{4}\left\|\nabla \delta \widehat{\mathbf{E}}^{n}\right\|_{0}^{2}+\frac{C}{\sigma^{n+1}}\left\|\nabla \widehat{\mathbf{E}}^{n}\right\|_{0}^{2}
$$


which leads to the improved bound

$$
\sum_{n=1}^{N} \sigma^{n+1} B_{2}^{n} \leq \frac{\mu \tau}{4} \sum_{n=1}^{N} \sigma^{n+1}\left\|\nabla \delta \widehat{\mathbf{E}}^{n}\right\|_{0}^{2}+C \sum_{n=1}^{N}\left\|\nabla \widehat{\mathbf{E}}^{n}\right\|_{0}^{2} \leq C+\frac{\mu \tau}{4} \sum_{n=1}^{N}\left\|\nabla \delta \widehat{\mathbf{E}}^{n}\right\|_{0}^{2}
$$

and proves the weighted estimate (5.24). We finally observe that if Assumption A4 is valid, then so is Lemma 3.5 thereby making unnecessary the use of weight $\sigma^{n+1}$ in (5.32) and (5.33). This leads to an inequality similar to (5.34) without weights and implies the asserted uniform estimate.

Remark 5.12 (Convergence rates for velocity of explicit Algorithm 1). If we treat convection explicitly, as written in (4.7), then we also need the customary but strong assumption (4.8) to obtain the results of Theorems 5.3 [5.7] and 5.10.

\section{ERROR ANALYSIS FOR ORESSURE}

The goal of this section is to estimate the pressure error in $L^{2}\left(0, T ; L^{2}(\Omega)\right)$ for Algorithm 11 where $p^{n+1}$ is computed according to (2.2), namely,

$$
p^{n+1}=-\frac{\phi^{n+1}-\phi^{n}}{\tau}+\mu \Delta \phi^{n+1} .
$$

This hinges on the error estimate for time derivative of velocity of Theorem 5.10,

Theorem 6.1 (Rate of convergence for pressure). If Assumptions A1-A 9 hold, then the pressure error function $e^{n+1}$ satisfies the weighted estimate

$$
\tau \sum_{n=0}^{N} \sigma^{n+1}\left\|e^{n+1}\right\|_{0}^{2} \leq C \tau .
$$

If Assumption A4 is also valid, then the following uniform error estimate holds:

$$
\tau \sum_{n=0}^{N}\left\|e^{n+1}\right\|_{0}^{2} \leq C \tau \text {. }
$$

Proof. We recall the existence of $\beta>0$ such that (inf-sup condition) [1 9

$$
\beta\|q\|_{0} \leq \sup _{\mathbf{w} \in b f H_{0}^{1}(\Omega)} \frac{\langle q, \operatorname{div} \mathbf{w}\rangle}{\|\nabla \mathbf{w}\|_{0}} \quad \forall q \in L_{0}^{2}(\Omega) .
$$

Consequently, it suffices to estimate $\left\langle e^{n+1}, \operatorname{div} \mathbf{w}\right\rangle$ in terms of $\|\nabla \mathbf{w}\|_{0}$. Multiplying (5.9) by $\mathrm{w}$ and utilizing (5.2) and (6.1), we end up with

$$
\begin{aligned}
\left\langle e^{n+1}, \operatorname{div} \mathbf{w}\right\rangle= & \left\langle\delta \mathbf{E}^{n+1}, \mathbf{w}\right\rangle+\mu\left\langle\nabla \widehat{\mathbf{E}}^{n+1}, \nabla \mathbf{w}\right\rangle \\
& +\left(\mathfrak{N}\left(\mathbf{u}\left(t^{n+1}\right), \mathbf{u}\left(t^{n+1}\right), \mathbf{w}\right)-\mathfrak{N}\left(\mathbf{u}^{n}, \widehat{\mathbf{u}}^{n+1}, \mathbf{w}\right)\right) \\
& -\mu\left\langle\Delta\left(\phi^{n+1}-\phi^{n}\right), \operatorname{div} \mathbf{w}\right\rangle-\left\langle\mathbf{R}^{n+1}, \mathbf{w}\right\rangle=\sum_{i=1}^{5} A_{i} .
\end{aligned}
$$

We now proceed to estimate each term $A_{1}$ to $A_{5}$ separately. We first note that

$$
A_{1} \leq C\left\|\delta \mathbf{E}^{n+1}\right\|_{0}\|\mathbf{w}\|_{0} \leq C\left\|\delta \mathbf{E}^{n+1}\right\|_{0}\|\nabla \mathbf{w}\|_{0}, \quad A_{2} \leq\left\|\nabla \widehat{\mathbf{E}}^{n+1}\right\|_{0}\|\nabla \mathbf{w}\|_{0} .
$$


Term $A_{3}$ can be dealt with, with the aid of (3.3) and Theorem 5.3 as follows:

$$
\begin{aligned}
A_{3}= & \mathfrak{N}\left(\mathbf{u}\left(t^{n+1}\right)-\mathbf{u}\left(t^{n}\right), \mathbf{u}\left(t^{n+1}\right), \mathbf{w}\right)+\mathfrak{N}\left(\mathbf{E}^{n}, \mathbf{u}\left(t^{n+1}\right), \mathbf{w}\right) \\
& -\mathfrak{N}\left(\mathbf{E}^{n}, \widehat{\mathbf{E}}^{n+1}, \mathbf{w}\right)+\mathfrak{N}\left(\mathbf{u}\left(t^{n}\right), \widehat{\mathbf{E}}^{n+1}, \mathbf{w}\right) \\
\leq & C\left(\left\|\mathbf{u}\left(t^{n+1}\right)-\mathbf{u}\left(t^{n}\right)\right\|_{0}\left\|\mathbf{u}\left(t^{n+1}\right)\right\|_{2}+\left\|\mathbf{E}^{n}\right\|_{0}\left\|\mathbf{u}\left(t^{n+1}\right)\right\|_{2}\right. \\
& \left.+\left\|\mathbf{E}^{n}\right\|_{1}\left\|\widehat{\mathbf{E}}^{n+1}\right\|_{1}+\left\|\mathbf{u}\left(t^{n}\right)\right\|_{2}\left\|\widehat{\mathbf{E}}^{n+1}\right\|_{1}\right)\|\nabla \mathbf{w}\|_{0} \\
\leq & C\left(\left\|\nabla \widehat{\mathbf{E}}^{n+1}\right\|_{0}+\left\|\mathbf{E}^{n}\right\|_{0}+\left\|\mathbf{u}\left(t^{n+1}\right)-\mathbf{u}\left(t^{n}\right)\right\|_{0}\right)\|\nabla \mathbf{w}\|_{0},
\end{aligned}
$$

because (5.6) and (5.7) imply $\left\|\mathbf{E}^{n}\right\|_{1} \leq C$. On the other hand, we have

$$
A_{4} \leq \mu\left\|\Delta\left(\phi^{n+1}-\phi^{n}\right)\right\|_{0}\|\nabla \mathbf{w}\|_{0}, \quad A_{5} \leq\left\|\mathbf{R}^{n+1}\right\|_{-1}\|\nabla \mathbf{w}\|_{0} .
$$

Inserting the estimates for $A_{1}$ to $A_{4}$ back into (6.5) and employing (6.4), we obtain

$$
\begin{aligned}
C\left\|e^{n+1}\right\|_{0} \leq & \left\|\delta \mathbf{E}^{n+1}\right\|_{0}+\left\|\nabla \widehat{\mathbf{E}}^{n+1}\right\|_{0}+\left\|\mathbf{E}^{n}\right\|_{0} \\
& +\left\|\mathbf{R}^{n+1}\right\|_{-1}+\left\|\Delta\left(\phi^{n+1}-\phi^{n}\right)\right\|_{0}+\left\|\mathbf{u}\left(t^{n+1}\right)-\mathbf{u}\left(t^{n}\right)\right\|_{0} .
\end{aligned}
$$

We now square, multiply by $\tau \sigma^{n+1}$ (resp. $\tau$ ), and sum over $n$ from 0 to $N$. Recalling (5.29) and 5.11) and invoking Lemma 3.4 Theorems 5.7 and 5.10 and Lemma 5.9 the assertion (6.2) (resp. 6.3) follows.

Remark 6.2 (Optimality). Both error estimates of Theorem 6.1 are optimal according to the regularity in time for pressure of Lemma 3.4, because the operator involved is not angle-bounded [16].

Remark 6.3 (Rate of convergence for pressure of explicit Algorithm 1). If Assumptions $\mathrm{A} 1-\mathrm{A} 3$ and (4.8) hold, then the pressure error functions $e^{n+1}$ of Algorithm[1 with explicit convection (2.4) satisfy (6.2). Moreover, they satisfy (6.3) provided Assumption $\mathrm{A} 4$ is also valid.

\section{The gauge method with Dirichlet condition}

In this section, we examine the Dirichlet condition (1.5) and thereby uncover a fundamental obstruction for computing pressure, which severely limits its applicability. The chief difficulty in deriving an error estimate for velocity is that now $\mathbf{u}^{n} \cdot \boldsymbol{\nu} \neq 0$. This is responsible for the reduced order $\mathcal{O}(\sqrt{\tau})$ of Theorem 7.3, which is consistent with the rate obtained in 26] via asymptotics, and for the additional but realistic regularity assumption (7.4).

Algorithm 1 can be modified as follows to account for (1.5):

Algorithm 2 (Gauge method with Dirichlet condition (1.5)). Start with initial values $\phi^{0}=0$ and $\mathbf{a}^{0}=\mathbf{u}^{0}=\mathbf{u}(0, \mathbf{x})$. Repeat for $1 \leq n \leq N$

Step 1: Find $\mathbf{a}^{n+1}$ as the solution of

$$
\begin{aligned}
\frac{\mathbf{a}^{n+1}-\mathbf{a}^{n}}{\tau}+\left(\mathbf{u}^{n} \cdot \nabla\right) \mathbf{a}^{n+1}+\left(\mathbf{u}^{n} \cdot \nabla\right) \nabla \phi^{n}-\mu \Delta \mathbf{a}^{n+1}=\mathbf{f}\left(t^{n+1}\right), & \text { in } \Omega, \\
\mathbf{a}^{n+1} \cdot \boldsymbol{\nu}=-\partial_{\boldsymbol{\nu}} \phi^{n}, \quad \mathbf{a}^{n+1} \cdot \boldsymbol{\tau}=0, & \text { on } \partial \Omega .
\end{aligned}
$$

Step 2: Find $\phi^{n+1}$ as the solution of

$$
\begin{aligned}
-\Delta \phi^{n+1} & =\operatorname{div} \mathbf{a}^{n+1}, & & \text { in } \Omega, \\
\phi^{n+1} & =0, & & \text { on } \partial \Omega .
\end{aligned}
$$


Step 3: Update $\mathbf{u}^{n+1}$ :

$$
\mathbf{u}^{n+1}=\mathbf{a}^{n+1}+\nabla \phi^{n+1}, \quad \text { in } \Omega .
$$

Remark 7.1 (Velocity boundary condition). In Algorithm [2, the boundary conditions of velocity $\mathbf{u}^{n+1}$ are

$$
\mathbf{u}^{n+1} \cdot \boldsymbol{\nu}=\partial_{\boldsymbol{\tau}} \psi^{n+1}=\partial_{\boldsymbol{\nu}} \phi^{n+1}-\partial_{\boldsymbol{\nu}} \phi^{n}, \quad \mathbf{u}^{n+1} \cdot \boldsymbol{\tau}=0, \quad \text { on } \partial \Omega .
$$

Remark 7.2 (Compatibility condition). Upon integrating both sides of (7.2) and using the boundary conditions of (7.1), we discover the relation

$$
\int_{\Omega} \Delta \phi^{n+1} d \mathbf{x}=\int_{\partial \Omega} \partial_{\boldsymbol{\nu}} \phi^{n+1} d \Gamma=-\int_{\partial \Omega} \mathbf{a}^{n+1} \cdot \boldsymbol{\nu} d \Gamma=\int_{\partial \Omega} \partial_{\boldsymbol{\nu}} \phi^{n} d \Gamma=\int_{\Omega} \Delta \phi^{n} d \mathbf{x}
$$

for Algorithm 2, This means that $\int_{\Omega} \Delta \phi^{n} d \mathbf{x}$ must be constant for all time steps $n$, which is not true in general. So, we cannot expect the numerical solution $\phi^{n}$ to converge to the exact solution $\phi$, as computations corroborate [15, 19]. Since pressure $p^{n}$ and $\phi^{n}$ are linked via (2.2), we cannot expect convergence of $p^{n}$ to $p$. Therefore, Algorithm 2 cannot be used for approximating $p$. Surprisingly, the velocity $\mathbf{u}^{n+1}$ converges to the exact solution $\mathbf{u}$ with a rate $\mathcal{O}(\sqrt{\tau})$ under realistic regularity assumptions, which are much weaker than those in [26] for a similar rate.

Theorem 7.3 (Reduced rate of convergence for velocity of Algorithm 21). Let Assumptions A]-A hold as well as

$$
\int_{0}^{T}\left\|p_{t}(t)\right\|_{0}^{2} d t \leq M
$$

Then the error functions of Algorithm $\mathbb{Q}$ satisfy

$$
\begin{aligned}
\left\|\mathbf{E}^{N+1}\right\|_{0}^{2} & +\frac{\mu \tau}{4} \sum_{n=0}^{N}\left\|\nabla \widehat{\mathbf{E}}^{n+1}\right\|_{0}^{2} \\
& +\sum_{n=0}^{N}\left(\left\|\mathbf{E}^{n+1}-\mathbf{E}^{n}\right\|_{0}^{2}+\left\|\nabla\left(\phi^{n+1}-\phi^{n}\right)\right\|_{0}^{2}\right) \leq C \tau .
\end{aligned}
$$

Proof. Since $\widehat{\mathbf{E}}^{n+1}=0$ on $\partial \Omega$ according to (5.1), the departing point is again (5.10). To estimate $A_{1}$ and $A_{4}$, we proceed as in Theorem 5.3 and thereby obtain (5.12) and (5.14), respectively. The remaining two terms $A_{2}$ and $A_{3}$ are more delicate and are handled together as follows:

$$
\begin{aligned}
A_{2}+A_{3}= & 2 \tau\left\langle p\left(t^{n+1}\right)-p\left(t^{n}\right), \Delta\left(\phi^{n+1}-\phi^{n}\right)\right\rangle \\
& -\frac{2}{\mu} \tau\left\langle q^{n}, q^{n+1}-q^{n}\right\rangle+\frac{2}{\mu} \tau\left\langle q^{n}, p\left(t^{n+1}\right)-p\left(t^{n}\right)\right\rangle=B_{1}+B_{2}+B_{3},
\end{aligned}
$$

where $q^{n}:=p\left(t^{n}\right)-\mu \Delta \phi^{n}$. We have $\left\|p\left(t^{n+1}\right)-p\left(t^{n}\right)\right\|_{0}^{2} \leq C \tau \int_{t^{n}}^{t^{n+1}}\left\|p_{t}(t)\right\|_{0}^{2} d t$, whence using Lemma 5.2

$$
B_{1} \leq \frac{C \tau^{2}}{\mu} \int_{t^{n}}^{t^{n+1}}\left\|p_{t}(t)\right\|_{0}^{2} d t+\frac{\mu \tau}{8}\left\|\nabla \widehat{\mathbf{E}}^{n+1}\right\|_{0}^{2} .
$$


For $B_{2}$ we employ the inequality $(a+b)^{2} \leq(1+\varepsilon) a^{2}+\left(1+\frac{1}{\varepsilon}\right) b^{2}$ for $\varepsilon=\frac{1}{8}$,

$$
\begin{aligned}
B_{2} & =-\frac{\tau}{\mu}\left(\left\|q^{n+1}\right\|_{0}^{2}-\left\|q^{n}\right\|_{0}^{2}\right)+\frac{\tau}{\mu}\left\|\left(p\left(t^{n+1}\right)-p\left(t^{n}\right)\right)-\mu \Delta\left(\phi^{n+1}-\phi^{n}\right)\right\|_{0}^{2} \\
& \leq-\frac{\tau}{\mu}\left(\left\|q^{n+1}\right\|_{0}^{2}-\left\|q^{n}\right\|_{0}^{2}\right)+\frac{C \tau^{2}}{\mu} \int_{t^{n}}^{t^{n+1}}\left\|p_{t}(t)\right\|_{0}^{2} d t+\frac{9 \mu \tau}{8}\left\|\nabla \widehat{\mathbf{E}}^{n+1}\right\|_{0}^{2} .
\end{aligned}
$$

Since

$$
B_{3} \leq \frac{C \tau^{2}}{\mu}\left\|q^{n}\right\|_{0}^{2}+\frac{C \tau}{\mu} \int_{t^{n}}^{t^{n+1}}\left\|p_{t}(t)\right\|_{0}^{2} d t
$$

inserting these expressions into (5.10) and summing over $n$ from 0 to $N$, yield

$$
\begin{aligned}
\left\|\mathbf{E}^{N+1}\right\|_{0}^{2} & +\sum_{n=0}^{N}\left(\left\|\mathbf{E}^{n+1}-\mathbf{E}^{n}\right\|_{0}^{2}+\left\|\nabla\left(\phi^{n+1}-\phi^{n}\right)\right\|_{0}^{2}+\frac{\mu \tau}{4}\left\|\nabla \widehat{\mathbf{E}}^{n+1}\right\|_{0}^{2}\right) \\
& +\frac{\tau}{\mu}\left\|q^{N+1}\right\|_{0}^{2} \leq \frac{C \tau}{\mu} \sum_{n=0}^{N}\left\|\mathbf{E}^{n+1}\right\|_{0}^{2}+\frac{C \tau^{2}}{\mu} \sum_{n=0}^{N}\left\|q^{n}\right\|_{0}^{2}+\frac{\tau}{\mu}\left\|p\left(t_{0}\right)\right\|_{0}^{2} \\
& +C \tau \int_{0}^{t^{N+1}}\left(\left\|p_{t}(t)\right\|_{0}^{2}+\sigma(t)\left\|\mathbf{u}_{t t}(t)\right\|_{0}^{2}+\tau\left\|\mathbf{u}_{t}(t)\right\|_{0}^{2}\right) d t
\end{aligned}
$$

Finally, the discrete Gronwall lemma implies (7.5) and concludes the proof.

Remark 7.4 (Reduced rate of convergence for velocity of explicit Algorithm 2). If Assumptions A(1-A3, (4.8), and (7.4) hold, then the error functions of Algorithm 2 with explicit convection (2.4) satisfy (7.5).

\section{REFERENCES}

[1] F. Brezzi and M. Fortin, Mixed and Hybrid Finite Element Methods, Springer-Verlag (1991). MR 92d:65187

[2] D.L. Brown, R. Cortez, and M.L. Minion, Accurate projection methods for the incompressible Navier-Stokes equations, J. Comput. Phys., 168 (2001) 464-499. MR 2002a:76112

[3] A.J. Chorin, Numerical solution of the Navier-Stokes equations, Math. Comp., 22 (1968) 745-762. MR 39:3723

[4] P. Constantin and C. Foias, Navier-Stokes Equations, The University of Chicago Press (1988). MR 90b:35190

[5] M. Dauge, Stationary Stokes and Navier-Stokes systems on two- or three-dimensional domains with corners, SIAM J. Math. Anal. 20 (1989), 74-97. MR 90b:35191

[6] W. E and J.-G. Liu, Gauge finite element method for incompressible flows, Int. J. Num. Meth. Fluids, 34 (2000), 701-710.

[7] W. E and J.-G. Liu, Gauge method for viscous incompressible flows, Comm. Math. Sci., 1 (2003), 317-332.

[8] W. E and J.-G. Liu, Projection method I: Convergence and numerical boundary layers, SIAM J. Numer. Anal., 32 (1995), 1017-1057. MR 96e:65061

[9] V. Girault, and P.A. Raviart, Finite Element Methods for Navier-Stokes Equations, SpringerVerlag (1986). MR 88b:65129

[10] C. Johnson, R. Rannacher, and M. Boman, Numerics and hydrodynamic stability: toward error control in computational fluid dynamics, SIAM J. Numer. Anal. 32 (1995), 1058-1079. MR 96j:76089

[11] J.G. Heywood and R. Rannacher, Finite element approximation of the non-stationary Navier-stokes problem. I. regularity of solutions and second-order error estimates for spatial discretization, SIAM J. Numer. Anal., 19 (1982), 275-311. MR 83d:65260

[12] R.B. Kellogg and J.E. Osborn, A regularity result for the stokes problems in a convex polygon, J. Funct. Anal., 21 (1976), 397-431. MR 53:8649 
[13] R.H. Nochetto and J.-H. Pyo, Optimal relaxation parameter for the Uzawa method Numer. Math. (to appear).

[14] R.H. Nochetto and J.-H. Pyo, A finite element Gauge-Uzawa method. Part I : the NavierStokes equations (to appear).

[15] R.H. Nochetto and J.-H. Pyo, Comparing Gauge-Uzawa methods with other projection methods (to appear).

[16] R.H. Nochetto, G. Savaré, and C. Verdi, A posteriori error estimates for variable time-step discretizations of nonlinear evolution equations, Comm. Pure Appl. Math., 53 (2000), 525589. MR 2000k:65142

[17] V.I. Oseledets, A new form of writing out the Navier-Stokes equation. The Hamiltonian formalism, Russian Math. Surveys, 44 (1989), 210-211. MR 91e:58173

[18] A. Prohl, Projection and Quasi-Compressibility Methods for Solving the Incompressible Navier-Stokes Equations, B.G.Teubner, Stuttgart (1997). MR 98k:65058

[19] J.-H. Pyo, The Gauge-Uzawa and related projection finite element methods for the evolution Navier-Stokes equations, Ph.D dissertation, University of Maryland (2002).

[20] R. Rannacher, On Chorin's projection method for the incompressible Navier-Stokes equations, Springer-Verlag, Lecture Notes in Mathematics 1530 (1992), 167-183. MR 95a:65149

[21] J. Shen, On error estimates of projection methods for Navier-Stokes equations: first order schemes, SIAM J. Numer. Anal., 29 (1992), 57-77. MR 92m:35213

[22] R. Temam, Navier-Stokes Equations and Nonlinear Functional Analysis, SIAM CBMS 66 (1995). MR 96e:35136

[23] R. Temam, Navier-Stokes Equations, North-Holland (1984). MR 86m:76003

[24] R. Temam, Sur l'approximation de la solution des equations de Navier-Stokes par la methode des pas fractionnaires. II, Arch. Rational Mech. Anal., 33 (1969), 377-385. MR 39:5968

[25] V. Thomèe, Galerkin Finite Element Methods for Parabolic Problems, Springer-Verlag (1997). MR 98m:65007

[26] C. Wang and J.-G. Liu, Convergence of gauge method for incompressible flow, Math. Comp., 69 (2000), 1385-1407. MR 2001a:65103

Department of Mathematics and Institute for Physical Science and Technology, University of Maryland, College Park, Maryland 20742

E-mail address: rhn@math.umd.edu

$U R L:$ http://www.math.umd.edu/ ${ }^{\sim} \mathrm{rhn}$

Department of Mathematics, Purdue University, West Lafayette, Indiana 47907

E-mail address: pjh@math.purdue.edu

$U R L:$ http://www.math.purdue.edu/ ${ }^{p j h}$ 